\title{
Article
}

\section{Boris computational spintronics-High performance multi-mesh magnetic and spin transport modeling software}

\author{
Lepadatu, Serban
}

Available at https://clok.uclan.ac.uk/36281/

Lepadatu, Serban orcid iconORCID: 0000-0001-6221-9727 (2020) Boris computational spintronics-High performance multi-mesh magnetic and spin transport modeling software. Journal of Applied Physics, 128 (24). p. 243902. ISSN 0021-8979

It is advisable to refer to the publisher's version if you intend to cite from the work.

http://dx.doi.org/10.1063/5.0024382

For more information about UCLan's research in this area go to http://www.uclan.ac.uk/researchgroups/ and search for <name of research Group>.

For information about Research generally at UCLan please go to http://www.uclan.ac.uk/research/

All outputs in CLoK are protected by Intellectual Property Rights law, including Copyright law. Copyright, IPR and Moral Rights for the works on this site are retained by the individual authors and/or other copyright owners. Terms and conditions for use of this material are defined in the policies page.

\section{CLoK}

Central Lancashire online Knowledge www.clok.uclan.ac.uk

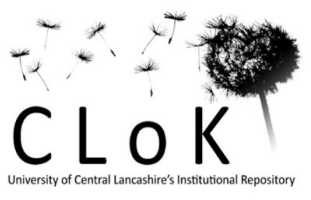




\section{Boris computational spintronics-High performance multi-mesh magnetic and spin transport modeling software ${ }^{\circ}$}

Cite as: J. Appl. Phys. 128, 243902 (2020); https://doi.org/10.1063/5.0024382

Submitted: 06 August 2020 . Accepted: 07 December 2020. Published Online: 28 December 2020

(D) Serban Lepadatu

\section{COLLECTIONS}

EP This paper was selected as an Editor's Pick
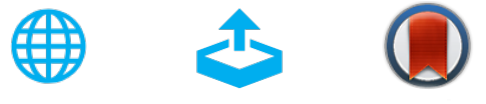

\section{ARTICLES YOU MAY BE INTERESTED IN}

A technique to measure spin-dependent trapping events at the metal-oxide-semiconductor field-effect transistor interface: Near zero field spin-dependent charge pumping Journal of Applied Physics 128, 244501 (2020); https://doi.org/10.1063/5.0027214

Reconfigurable and self-biased magnonic metamaterials

Journal of Applied Physics 128, 240902 (2020); https://doi.org/10.1063/5.0033254

THz-range Faraday rotation in the Weyl semimetal candidate $\mathrm{CO}_{2} \mathrm{TiGe}$

Journal of Applied Physics 128, 244303 (2020); https://doi.org/10.1063/5.0033536

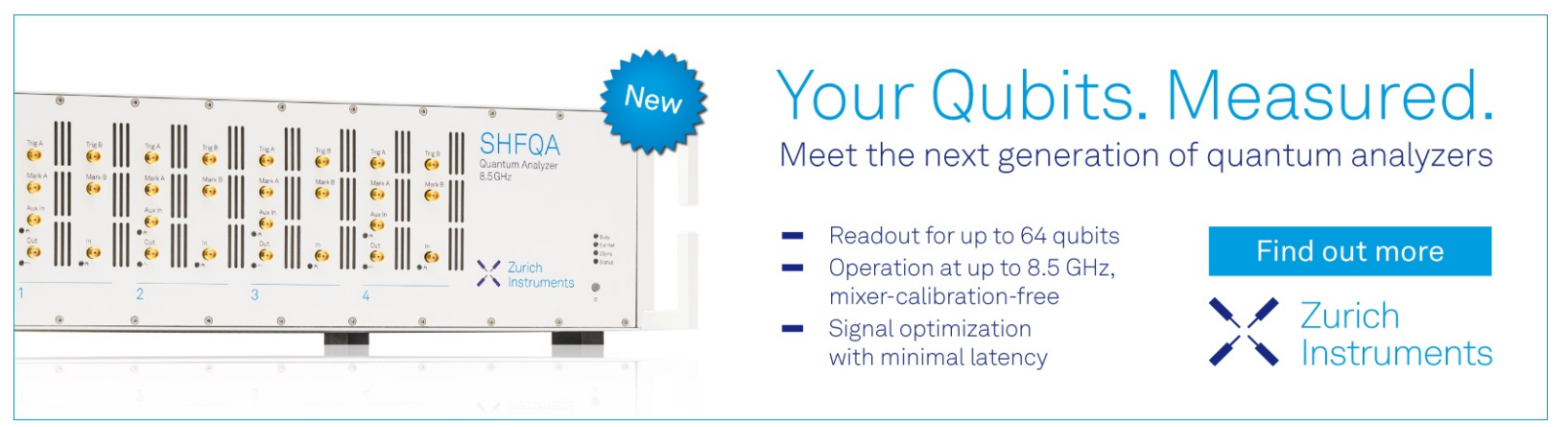




\title{
Boris computational spintronics-High performance multi-mesh magnetic and spin transport modeling software
}

Cite as: J. Appl. Phys. 128, 243902 (2020); doi: 10.1063/5.0024382

Submitted: 6 August 2020 - Accepted: 7 December 2020 .

Published Online: 28 December 2020

Serban Lepadatu ${ }^{\text {a) }}$ (D)

\begin{abstract}
AFFILIATIONS
Jeremiah Horrocks Institute for Mathematics, Physics and Astronomy, University of Central Lancashire, Preston PR1 2HE, United Kingdom
\end{abstract}

a) Author to whom correspondence should be addressed: SLepadatu@uclan.ac.uk

\begin{abstract}
This work discusses the design and testing of a new computational spintronics research software. Boris is a comprehensive multi-physics open-source software, combining micromagnetics modeling capabilities with drift-diffusion spin transport modeling and a heat flow solver in multi-material structures. A multi-mesh paradigm is employed, allowing modeling of complex multi-layered structures with independent discretization and arbitrary relative positioning between different computational meshes. Implemented micromagnetics models include not only ferromagnetic materials modeling, but also two-sublattice models, allowing simulations of antiferromagnetic and ferrimagnetic materials, fully integrated into the multi-mesh and multi-material design approach. High computational performance is an important design consideration in Boris, and all computational routines can be executed on graphical processing units (GPUs), in addition to central processing units. In particular, a modified 3D convolution algorithm is used to compute the demagnetizing field on the GPU, termed pipelined convolution, and benchmark comparisons with existing GPU-accelerated software Mumax3 have shown performance improvements up to twice faster.
\end{abstract}

Published under license by AIP Publishing. https://doi.org/10.1063/5.0024382

\section{OVERVIEW}

Micromagnetics is a field of study concerned with understanding magnetization processes on the continuum scale and is an invaluable tool in interpreting experimental results, designing spintronics devices, testing analytical methods, and predicting new effects. Existing micromagnetics software includes open-source finite difference packages OOMMF, ${ }^{1}$ Mumax $3,{ }^{2}$ and Fidimag. ${ }^{3}$ A number of other micromagnetics packages are also available, including finite element/boundary element methods, both opensource and commercial, with a review given in Ref. 4 .

Boris is a micromagnetics-oriented multi-physics research software. In contrast to the existing finite difference packages, it is specifically designed as a multi-mesh and multi-material software. Arbitrary geometries can be handled, where long-range interactions such as the magnetostatic interaction and Oersted field are calculated across all relevant computational meshes, and short-range interactions between neighboring meshes are treated using appropriate composite media boundary conditions. An overview is given in Fig. 1. Magnetization dynamics are computed using the Landau-Lifshitz-Gilbert (LLG) equation ${ }^{5}$ or the Landau-LifshitzBloch (LLB) equation, ${ }^{6}$ either of which may be augmented by thermal fluctuations (stochastic versions), ${ }^{7,8}$ Zhang-Li spin transfer torques (STTs), ${ }^{9}$ interfacial STT (ISTT), ${ }^{10,11}$ spin-orbit torques (SOTs) due to the spin-Hall effect (SHE), ${ }^{12}$ Slonczewski spin torques, ${ }^{13}$ or spin torques computed self-consistently using a spin transport solver. The spin transport solver is based on a driftdiffusion model with circuit theory boundary conditions ${ }^{14-16}$ and self-consistently calculates charge currents, spin currents, and spin accumulations in multi-layer structures. ${ }^{17}$ In addition to obtaining spin torques self-consistently, several effects may be computed, including anisotropic magneto-resistance (AMR), current perpendicular to plane giant magneto-resistance (CPP-GMR), ${ }^{15} \mathrm{SHE}$ and inverse SHE (ISHE), ${ }^{18}$ spin pumping, ${ }^{19}$ charge pumping, and the topological Hall effect. ${ }^{20,21}$ A heat solver is also available, allowing calculation of heat flow in response to ambient conditions as well 


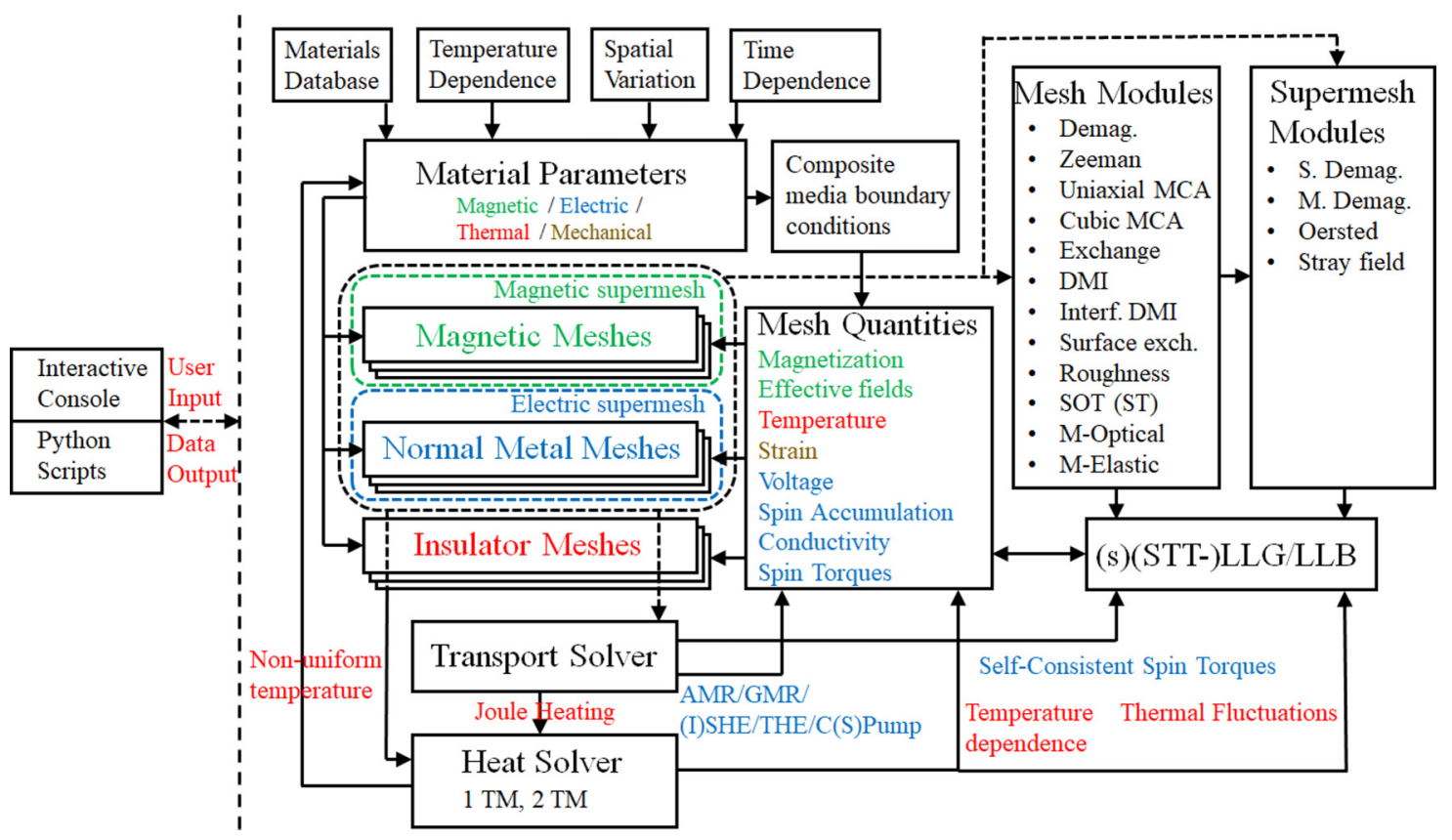

FIG. 1. Overview of computational information flow for Boris. Different computational mesh types may be configured, including ferromagnetic, antiferromagnetic, ferrimagnetic, normal metal, and insulator meshes. Each mesh has several computational modules available, including the transport and heat solvers. Supermeshes are the smallest rectangles encompassing all the individual meshes of the same type, with specific computational modules available. The information generated is used by an assigned magnetization dynamics equation (LLG/LLB) to evolve the magnetization data in the individual magnetic meshes.

as sources and sinks. An important source of heat is due to Joule heating from a current density calculated using the transport solver. This allows inclusion of temperature-dependent effects in the magnetization dynamics, including AMR-generated magnonic spin-Seebeck effect. ${ }^{22}$ Another heat source is due to ultrafast laser pulses, and a two-temperature model (2TM) is included to allow simulations of ultrafast demagnetization and recovery processes. ${ }^{23}$ Additionally, a two-sublattice model is implemented, allowing simulations of antiferromagnetic and ferrimagnetic materials, fully integrated within the multi-mesh computational paradigm, allowing, for example, simulations with exchange bias. All parameters appearing in the working equations are available as usercontrollable material parameters and may be assigned a temperature dependence, spatial variation, and time dependence; several spatial variation generators are available, including Voronoi tessellations, as well as user-defined dependences through mathematical equations or data files.

The computational meshes can be sized and discretized independently. One of the most difficult interactions to compute across several independent computational meshes is the magnetostatic interaction. A newly developed method, termed multi-layered convolution, ${ }^{24}$ allows computation of demagnetizing fields for multiple meshes with arbitrary thicknesses, arbitrary relative positioning and spacings, without impacting on the computational performance. Other long-range interactions include the Oersted field, which is computed from the current density obtained using the transport solver, as well as stray field computation from a number of fixed magnetic dipoles. Individual magnetic mesh modules include magneto-crystalline anisotropy (MCA), either uniaxial or cubic, direct exchange interaction, Dzyaloshinsky-Moriya interaction (DMI), ${ }^{25,26}$ either bulk or interfacial, surface exchange coupling, ${ }^{27}$ topographical surface, and edge roughness. ${ }^{28}$

The software has a modular structure and is open-source, ${ }^{29}$ facilitating community contributions of new computational modules. An extensive user manual ${ }^{30}$ is included, together with many examples of both scripted simulations, as well as preconfigured simulation setups. The software is provided with a graphical user interface for interactive display of simulation data, with user control enabled through a graphical console allowing intuitive and interactive control of simulations. The software may also be controlled using Python scripts, which communicate with Boris through network sockets, thus allowing either local or remote user control. The software has been programmed mainly in $\mathrm{C}++17$ and CUDA C, as well as Python. All computational routines can be executed on central processing units (CPUs), as well as graphical processing units (GPUs) using the CUDA framework. ${ }^{31}$ Supported operating systems include Windows 7, Windows 10, and Linux-based distributions; in the current version (2.9) Linux compilations of Boris do not include a graphical interface, only providing a basic text console; however, the software is otherwise fully functional and may be conveniently controlled using Python scripts, which is especially useful for controlling multiple 
independent instances in a Linux cluster. The code-base size currently consists of $\sim 130 \mathrm{k}$ source lines of code (comments and trivial lines excluded) and is contained in $\sim 800$ source code files, including a purpose-written object-oriented finite difference vector calculus library for both CPU and GPU computations. External dependences include FFTW $3^{32}$ and CUDA. ${ }^{31}$ Material definitions are made available through an online database of material parameters. $^{33}$ The online materials database allows users to contribute new entries through a set of simple built-in protocols described in the manual. Material definitions used in this work are given in the online materials database. ${ }^{33}$ Moreover, all the simulation scripts and files used to obtain the results presented here have been included in the Boris GitHub repository. ${ }^{29}$

\section{BASIC MICROMAGNETICS MODELING}

In the continuum approximation, magnetization dynamics may be computed using the LLG equation,

$$
\frac{\partial \mathbf{m}}{\partial t}=-\gamma \mathbf{m} \times \mathbf{H}_{e f f}+\alpha \mathbf{m} \times \frac{\partial \mathbf{m}}{\partial t} .
$$

Here, $\mathbf{m}$ is the normalized magnetization direction; $\gamma=\mu_{0} g_{\text {rel }}\left|\gamma_{e}\right|$, where $\gamma_{e}=-g \mu_{B} / \hbar$ is the electron gyromagnetic ratio and $g_{\text {rel }}$ is a relative gyromagnetic factor; $\alpha$ is the Gilbert damping factor, ${ }^{34}$ and $\mathbf{H}_{\text {eff }}$ is an effective field that includes a number of interactions as additive field contributions. In a basic micromagnetics formulation, these include an applied field contribution, the magnetostatic or demagnetizing field interaction, and the direct exchange interaction. Depending on the material simulated, a magneto-crystalline anisotropy contribution may be included, either uniaxial or cubic, as well as bulk or interfacial DMI. ${ }^{25,26}$ Equations for these contributions implemented in Boris are given in Appendix A. A number of evaluation methods are available for the magnetization dynamics equations. These are the fixed step methods Euler (1st order), trapezoidal Euler (2nd order), and Runge-Kuta (RK4-4th order). Adaptive time step methods are the adaptive Heun (2nd order), the multi-step AdamsBashforth-Moulton (2nd order), Runge-Kutta-Bogacki-Shampine (RK23-3rd order with embedded 2nd order error estimator), Runge-Kutta-Fehlberg (RKF45-4th order with embedded 5th order error estimator), Runge-Kutta-Cash-Karp (RKCK45-4th order with embedded 5th order error estimator), and RungeKutta-Dormand-Prince (RKDP54-5th order with embedded 4th order error estimator). For static problems, the steepest descent solver is available using Barzilai-Borwein stepsize selection formulas. $^{35,36}$

A widely used test for the validity and accuracy of LLG solvers is the $\mu$ MAG Standard Problem \#4. ${ }^{37}$ Here, the magnetization response to a magnetic field is computed for a $\mathrm{Ni}_{80} \mathrm{Fe}_{20}$ rectangle with dimensions $500 \times 125 \times 3 \mathrm{~nm}^{3}$, starting from a relaxed S-state. This is used as a test for correct implementation of the LLG equation and associated effective field terms (demagnetizing field, exchange interaction, and Zeeman term) as any errors result in significant deviations of the magnetization time dependence from accepted solutions. The results for the specified field $1\left(\mu_{0} H_{\mathrm{x}}\right.$ $=-24.6 \mathrm{mT}, \mu_{0} H_{\mathrm{y}}=4.3 \mathrm{mT}, \mu_{0} H_{\mathrm{z}}=0.0 \mathrm{mT}$ ) are shown in Fig. 2,

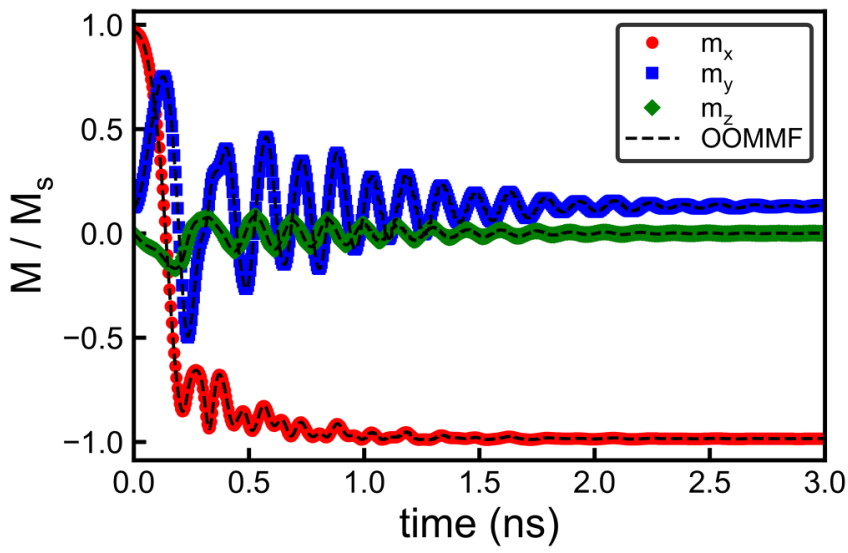

FIG. 2. Magnetization response computed for $\mu \mathrm{MAG}$ Standard Problem \#4 using Field 1 specification, showing the normalized components of magnetization, compared to the magnetization response computed in OOMMF. Overall $R^{2}$ measure of 0.999 was obtained.

compared with the results obtained using OOMMF. ${ }^{1}$ Excellent agreement throughout the switching process is obtained, with an overall $R^{2}$ measure between the two data sets of 0.999 . A cellsize of $5 \mathrm{~nm}$ was used here, which is consistent with the exchange length in $\mathrm{Ni}_{80} \mathrm{Fe}_{20}, l_{\text {ex }}=\sqrt{2 A / \mu_{0} M_{S}^{2}} \cong 5.7 \mathrm{~nm}$; however, Boris has been extensively tested using this problem with cellsize values down to $1 \mathrm{~nm}$, thus including both 2D and 3D modes. Similarly, the specified field $2\left(\mu_{0} H_{\mathrm{x}}=-35.5 \mathrm{mT}, \mu_{0} H_{\mathrm{y}}=-6.3 \mathrm{mT}, \mu_{0} H_{\mathrm{z}}=0.0 \mathrm{mT}\right)$ was also tested. The results in Fig. 2 were computed using the RK4 method with a fixed time step of $500 \mathrm{fs}$; however, all the implemented evaluation methods were successfully tested using this problem, for both CPU and GPU computations in single and double floating point precision.

A further test that requires a more advanced external field stimulus consists in computing the spin wave dispersion as described in Ref. 38. Here, a $\mathrm{Ni}_{80} \mathrm{Fe}_{20}$ magnonic waveguide track with $1 \mu \mathrm{m}$ length, $50 \mathrm{~nm}$ width, and $1 \mathrm{~nm}$ thickness is used, and spin waves are excited using a field pulse given by $H(t)=H_{\mathrm{e}}$ sinc $\left[k_{C}\left(x-x_{0}\right)\right] \operatorname{sinc}\left[k_{C}\left(y-x_{0}\right)\right] \operatorname{sinc}\left[2 \pi f_{C}\left(t-t_{0}\right)\right]$. Boris has a provision for input stimuli specification using mathematical formulas, simultaneously allowing spatial and temporal dependences. Here, the excitation field amplitude was set to $H_{\mathrm{e}}=400 \mathrm{kA} / \mathrm{m}$, frequency cutoff $f_{c}=500 \mathrm{GHz}$, and wave-vector cutoff $k_{C}=2 \pi \times 0.1255 \mathrm{rad} /$ $\mathrm{nm}$, as specified in Ref. 38. Using the Nyquist criterion, a time sampling interval of $1 \mathrm{ps}$ and a spatial sampling interval of $4 \mathrm{~nm}$ along the $1 \mu \mathrm{m}$ long track were used. The excitation was applied in the center of the track for a duration of $2 \mathrm{t}_{0}$, with a temporal sinc pulse center $t_{0}=200 \mathrm{ps}$. Three spin wave geometries are possible, depending on the direction of the bias field, namely, (i) backward volume for the bias field along the length, (ii) forward volume for the bias field along the thickness, and (iii) surface spin waves for the bias field along the width. The wave-vector direction for this problem in all cases is along the length of the track, which is determined by the spatial sampling direction. Results for the backward 
volume are shown in Fig. 3 for a damping value $\alpha=0.01$, where a bias field $H_{0}=804 \mathrm{kA} / \mathrm{m}$ was used, with the excitation field pulse applied along the width. The spin wave dispersion was obtained using a 2D Fourier transform from the y component of magnetization. The discretization cellsize was set to $1 \times 2 \times 1 \mathrm{~nm}^{3}$, with periodic boundary conditions ${ }^{39}$ used along the length only, and the RK4 method was used with a $50 \mathrm{fs}$ time step. This results in excellent agreement between the computed spin wave dispersion and the analytical dotted lines given by $w=w_{n}+2 \gamma A k^{2} / \mu_{0} M_{S}$; here, $w_{n}$ are the resonance frequencies obtained at $k=0 \mathrm{rad} / \mathrm{m}$. Similar tests were performed for the two remaining spin wave geometries.

The LLG equation may be modified to include STT and, in particular, Boris implements Zhang-Li STT, ${ }^{9,40}$ which is given by

$$
\frac{\partial \mathbf{m}}{\partial t}=-\gamma \mathbf{m} \times \mathbf{H}+\alpha \mathbf{m} \times \frac{\partial \mathbf{m}}{\partial t}+(\mathbf{u} \cdot \nabla) \mathbf{m}-\beta \mathbf{m} \times(\mathbf{u} \cdot \nabla) \mathbf{m} .
$$

The spin-drift velocity $\mathbf{u}$ is given by

$$
\mathbf{u}=\mathbf{J} \frac{P g \mu_{B}}{2 e M_{s}} \frac{1}{1+\beta^{2}}
$$

where $\mathbf{J}$ is the charge current density, $P$ is the current spin polarization, and $\beta$ is the non-adiabaticity parameter. The LLG-STT equation is widely used for studying the effect of bulk STT on magnetization textures, including transverse domain walls, Bloch and Néel domain walls, vortices, and skyrmions. In particular, domain wall velocity dependence on spin drift velocity may be computed, including simulation of Walker breakdown phenomenon. Since this is a very common type of computation Boris implements a moving domain wall algorithm, which allows efficient simulation of domain wall movement using a finite track length. End magnetic charges are removed using the stray field computed from magnetic dipoles at each end, and spin waves are absorbed by

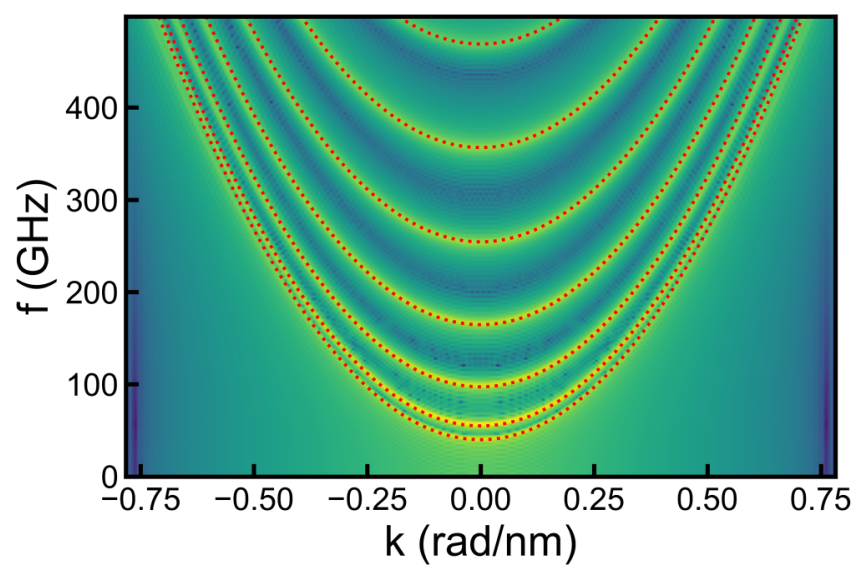

FIG. 3. Spin wave dispersion spectrum computed for the problem specified in Ref. 38, using a damping of 0.01 . The dotted lines are obtained from the formula $w=w_{n}+2 \gamma A k^{2} / \mu_{0} M_{S}$, with $w_{n}$ being the resonance frequencies obtained at $k=0 \mathrm{rad} / \mathrm{m}$. freezing the magnetization spins at the track ends. The domain wall is kept centered in the track and any domain wall displacement is recorded in a dedicated output parameter. This algorithm was tested previously, ${ }^{41}$ and it is straightforward to verify the expected relation $v / u=\beta / \alpha,{ }^{42}$ with $\mathrm{v}$ being the domain wall velocity far from Walker breakdown. Here, we show another test of the LLG-STT equation, based on the $\mu \mathrm{MAG}$ Standard Problem \#5. ${ }^{43}$ A permalloy rectangle with dimensions $100 \times 100 \times 10 \mathrm{~nm}^{3}$ is initialized with a vortex structure-Fig. 4(a) - and a constant current density resulting in a spin drift velocity $\mathrm{u}=-72.35 \mathrm{~m} / \mathrm{s}$ is applied for a range of non-adiabaticity parameter values. Results for $\beta=0.1$ are shown in Fig. 4(b), using a cubic cellsize of $2.5 \mathrm{~nm}$, plotting the $\mathrm{x}$ and $\mathrm{y}$ components of magnetization as a function of time. Excellent agreement with results computed in OOMMF is obtained, with an overall $R^{2}$ measure between the two data sets of 0.999 . Similar successful tests were performed for the remaining specified values of $\beta=0,0.05$, and 0.5 .

As a further example, we apply the micromagnetics model to compute the vertices population in large-scale simulations of square artificial spin ice (ASI), as studied experimentally in previous studies. ${ }^{44,45}$ A part of the simulated ASI array is shown in Fig. 5(a), where the $\mathrm{Ni}_{80} \mathrm{Fe}_{20}$ islands have dimensions

(a)
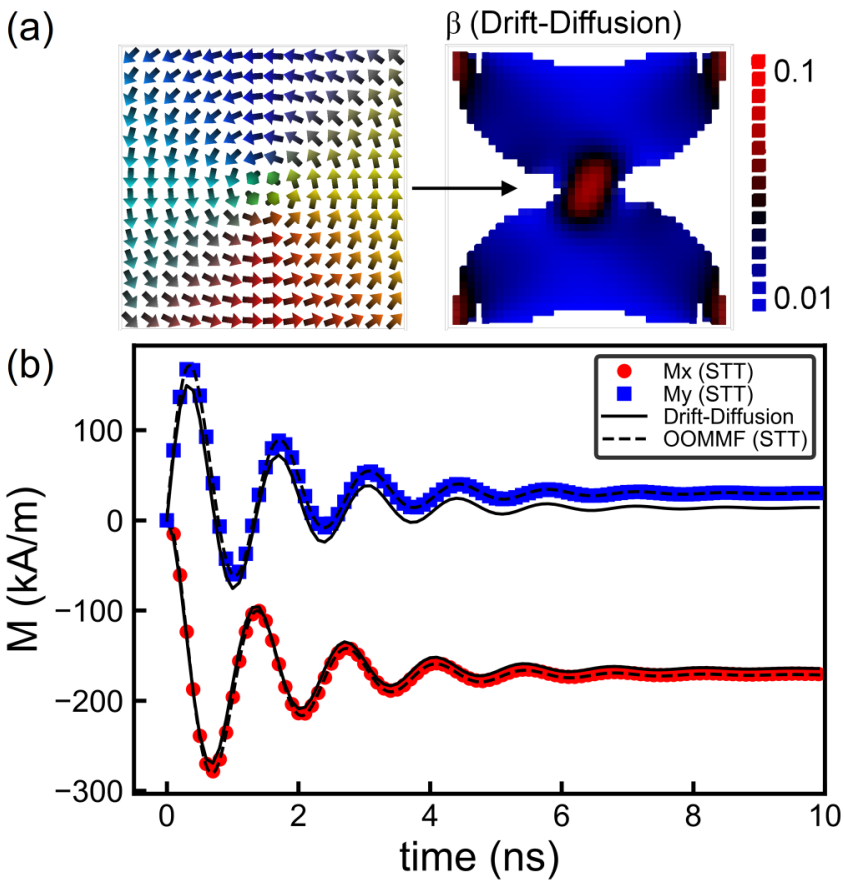

FIG. 4. Vortex dynamics computed for $\mu$ MAG Standard Problem $\# 5$ for $\beta=0.1$. (a) Relaxed starting vortex state, also showing the fitted spatial dependence of non-adiabaticity parameter computed using the spin transport drift-diffusion solver. (b) Vortex dynamics are shown for the LLG-STT solver with constant non-adiabaticity, compared to results computed in OOMMF. Overall $R^{2}$ measure of 0.999 was obtained. Results obtained using the self-consistent bulk spin torque obtained from the drift-diffusion model, where $\beta$ is no longer constant but varies due to in-plane spin diffusion, are also shown for comparison. 
(a)
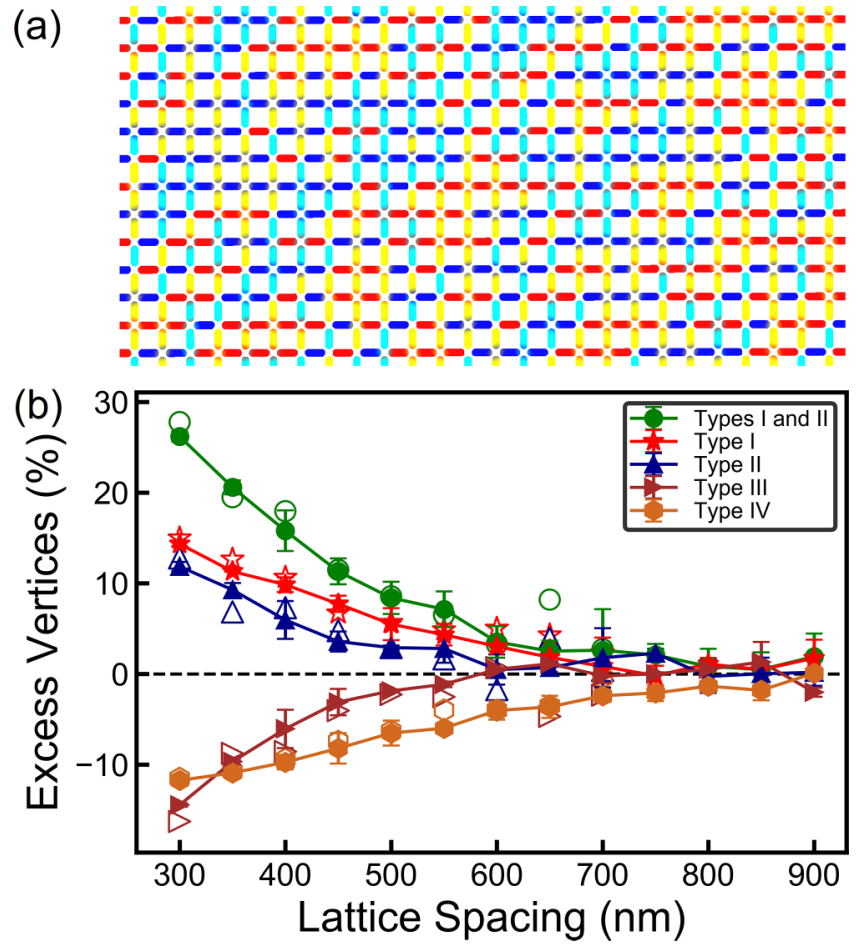

FIG. 5. Square ASI simulations with $220 \times 80 \times 25 \mathrm{~nm}^{3} \mathrm{Ni}_{80} \mathrm{Fe}_{20}$ elements, computed in both a 2D approximation and a 3D model, with in-plane periodic boundary conditions. For the $2 \mathrm{D}$ approximation, a maximum mesh size of $24 \mu \mathrm{m} \times 24 \mu \mathrm{m} \times 25 \mathrm{~nm}$ was used with a $5 \mathrm{~nm}$ in-plane cellsize (up to $23 \mathrm{M} \mathrm{sim}$ ulation cells). For the 3D model, a maximum mesh size of $6 \mu \mathrm{m} \times 6 \mu \mathrm{m} \times 25 \mathrm{~nm}$ was used with a $2.5 \times 2.5 \times 5 \mathrm{~nm}^{3}$ cellsize (up to $28.8 \mathrm{M}$ simulation cells). (a) Example part of simulated artificial spin ice array, with island direction color coded as blue: left, red: right, yellow: up, and cyan: down. (b) Calculated excess vertices for each vertex type as a function of lattice spacing, averaged over two simulation runs, comparable to results in Ref. 44. Solid symbols are used for the $2 \mathrm{D}$ approximation, and open symbols for the $3 \mathrm{D}$ model.

$220 \times 80 \times 25 \mathrm{~nm}^{3}$, as used in Ref. 44 . Due to the strong shape anisotropy and small island size, the magnetization is mostly in a single domain state, aligned to the longitudinal direction. Here, we distinguish four types of vertices, types I-IV, depending on the magnetization directions at each vertex, defined in Refs. 44 and 45. Due to magnetic frustration arising from dipolar interactions, the different vertex types have different energies-in particular, vertex types I and II have lower dipolar energy compared to vertex types III and IV, where particularly vertex type IV (all magnetization directions point in or all out) has a prohibitively large dipolar energy. Thus, compared to the expected random vertex population distribution in the absence of dipolar interactions $(12.5 \%, 25 \%$, $50 \%$, and $12.5 \%$, respectively), we expect to obtain an excess vertex population, dependent on the lattice spacing. Here, we compute this, by relaxation from a thermally demagnetized state, as a function of lattice spacing between $300 \mathrm{~nm}$ and $900 \mathrm{~nm}$, using both a $2 \mathrm{D}$ approximation and a $3 \mathrm{D}$ model, shown in Fig. 5(b). These results are in good agreement with those obtained experimentally, ${ }^{44}$ where increasing the lattice spacing causes the excess vertex populations to converge toward zero as the effect of dipolar interactions is reduced. These simulations have been completed on a GPU, automated using a single Python script available in the GitHub repository. ${ }^{29}$

\section{MULTI-MESH MICROMAGNETICS MODELING}

Due to progress in experimental magnetism increasingly, devices are composed of complex multi-layered structures, including multi-layered stacks used to study skyrmions ${ }^{46-49}$ and synthetic anti-ferromagnetic structures. ${ }^{50-52}$ Such multi-layered structures, which cannot be discretized effectively using a single uniform finite difference mesh, are difficult to study using software packages which only implement a single computational mesh without introducing approximations or using unnecessarily small cellsize values. In Boris, a multi-mesh paradigm has been adopted from the outset, allowing computations using multiple meshes that can be arbitrarily positioned relative to each other and with independent discretization cellsize values. Thus, while still benefiting from computationally efficient finite difference discretization, multilayered structures commonly found in experimental studies may be simulated without compromising accuracy or computational speed. This is accomplished using a new multi-layered convolution algorithm introduced in Boris, ${ }^{24}$ which is used to compute demagnetizing fields for a collection of finite difference computational meshes. For a collection of meshes $V_{k}(k=1, \ldots, n)$, the convolution sum may be written as

$$
\begin{gathered}
\mathbf{H}\left(\mathbf{r}^{\prime}{ }_{k l}\right)=-\sum_{\substack{i=1, \ldots, n \\
\mathbf{r}_{i j} \in V_{i}}} \mathbf{N}\left(\mathbf{r}_{k l}^{\prime}-\mathbf{r}_{i j}, \mathbf{h}_{k}, \mathbf{h}_{i}\right) \mathbf{M}\left(\mathbf{r}_{i j}\right), \\
k=1, \ldots, n ; \quad \mathbf{r}_{k l}^{\prime} \in V_{k} .
\end{gathered}
$$

Here, $\mathbf{r}_{i j}$ is the cell-centered position vector of cell $j$ in mesh $i$ $(i=1, \ldots, n)$, and $\mathbf{N}$ are inter- and intra-mesh demagnetizing tensors generalized from the Newell et al. formulas ${ }^{53}$ in Ref. 24. With a single computational mesh, the usual approach to efficiently evaluate the convolution sum is to use the convolution theorem, which involves computing the forward Fourier transform of the magnetization, multiplying point-by-point with the Fourier transform of the demagnetizing tensor (kernel) in the transform space, and finally taking the inverse Fourier transform to obtain the demagnetizing field. With multiple input meshes, a similar approach may be taken to evaluate Eq. (4), with summation of inter-mesh contributions moved to the transform space. Full details, including validation tests, are given in Ref. 24. Here, we extend this algorithm to use periodic boundary conditions based on the multiple images method $^{54}$ and demonstrate the use of multi-layered convolution by simulating the hysteresis loop in a $\left[\mathrm{Co}_{90} \mathrm{Fe}_{10}(4.9 \mathrm{~nm}) / \mathrm{Ru}(0.6 \mathrm{~nm}) / \mathrm{Co}_{90} \mathrm{Fe}_{10}(2.9 \mathrm{~nm}) / \mathrm{Ru}(0.6 \mathrm{~nm})\right]_{10}$ synthetic ferrimagnetic structure. Here, the layers preferentially align antiparallel due to RKKY interaction, ${ }^{55-57}$ as well as due to the magnetostatic field interaction between the layers. For two magnetic layers separated by a metallic spacer, the surface exchange 
energy density and effective field are given as

$$
\begin{aligned}
& \boldsymbol{\varepsilon}=-\frac{J_{1}}{\Delta} \mathbf{m}_{i} \cdot \mathbf{m}_{j}-\frac{J_{2}}{\Delta}\left(\mathbf{m}_{i} \cdot \mathbf{m}_{j}\right)^{2} \\
& \mathbf{H}_{i}=\frac{J_{1}}{\mu_{0} M_{S} \Delta} \mathbf{m}_{j}+\frac{2 J_{2}}{\mu_{0} M_{S} \Delta}\left(\mathbf{m}_{i} \cdot \mathbf{m}_{j}\right) \mathbf{m}_{j} .
\end{aligned}
$$

Here, $J_{1}$ and $J_{2}$ are the bilinear and biquadratic surface exchange coupling constants, respectively, with coupled magnetic moment directions given by $\mathbf{m}_{i}$ and $\mathbf{m}_{j}$, and $\Delta$ is the thickness of the ferromagnetic layer for which the $\mathbf{H}_{i}$ effective field contribution is calculated. The simulated structure is shown in the inset of Fig. 6, where we have used material parameters determined experimentally in Ref. 50 also available in the materials database ${ }^{33}$ and, in particular, $J_{1}=-1 \mathrm{~mJ} / \mathrm{m}^{2}$ with no biquadratic contribution. The simulated stack uses in-plane periodic boundary conditions, both for the differential operators-exchange field-and for the FFT-based convolution evaluation of the demagnetizing field. The simulated area is $300 \mathrm{~nm}^{2}$ with an in-plane cellsize of $3 \mathrm{~nm}$. A polycrystalline structure has been generated using in-plane Voronoi tessellation with $20 \mathrm{~nm}$ crystallites, where the uniaxial anisotropy easy axis varies randomly by $\pm 20^{\circ}$ around the $\mathrm{x}$ axis between the different crystallites. Results are shown in Fig. 6 with the field applied along the $\mathrm{x}$ axis direction. With a large external field, the magnetization in all the layers aligns along the field. Reduction of the field results in gradual rotation of the 10 thinner layers against the field direction, thus reducing the total energy as both the surface exchange and magnetostatic interactions result in preferentially anti-parallel alignment. As the field direction is reversed, all the thicker $\mathrm{Co}_{90} \mathrm{Fe}_{10}$ layers switch at once, with the thinner $\mathrm{Co}_{90} \mathrm{Fe}_{10}$ layer switching against the field due to the strong antiferromagnetic surface exchange interaction; further increasing the field results in gradual rotation of the 10 thinner layers toward the applied field direction. It should be noted that such a structure is very difficult to simulate using a single uniform finite difference computational mesh without introducing approximations, such as rounding the

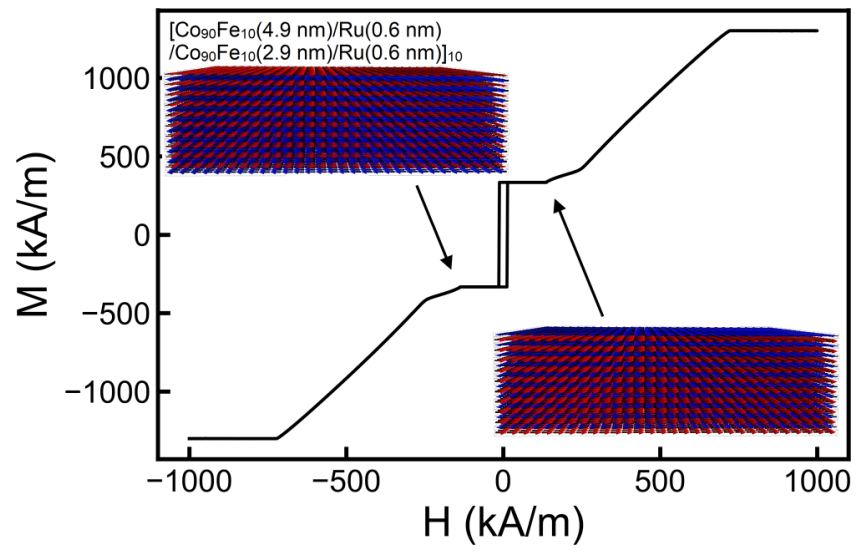

FIG. 6. Hysteresis loop in a synthetic ferrimagnetic 10-repetition multi-layered stack of $\left[\mathrm{CO}_{90} \mathrm{Fe}_{10}(4.9 \mathrm{~nm}) / \mathrm{Ru}(0.6 \mathrm{~nm}) / \mathrm{Co}_{90} \mathrm{Fe}_{10}(2.9 \mathrm{~nm}) / \mathrm{Ru}(0.6 \mathrm{~nm})\right]_{10}$. layer thicknesses, which become increasingly inaccurate as the number of repetitions increases. Even with the layer thickness rounded, for example, to $\mathrm{Co}_{90} \mathrm{Fe}_{10}(5 \mathrm{~nm}) / \mathrm{Ru}(1 \mathrm{~nm}) / \mathrm{Co}_{90} \mathrm{Fe}_{10}$ $(3 \mathrm{~nm})$, a discretization cellsize of $1 \mathrm{~nm}$ is still required along the $\mathrm{z}$ direction. With the multi-layered convolution algorithm in Boris, each layer can be considered a $2 \mathrm{D}$ mesh, rendering such simulations relatively trivial.

Further extensions to the micromagnetics model include topographical roughness and staircase corrections for the demagnetizing field, detailed previously ${ }^{28}$ and tested experimentally, ${ }^{58-60}$ as well as magnetoelastic contributions. ${ }^{61,62}$ Finally, all the material parameters included in simulations may be assigned spatial and temporal dependences through user-supplied mathematical formulas, input data files, or built-in generators. This allows simulations using polycrystalline or granular structures, as well as material defects and impurities.

\section{TRANSPORT SOLVER}

Inclusion of spin torques in modern micromagnetics solvers is an essential requirement, allowing modeling the effect of spin transfer torques on domain walls and skyrmions, spin-torque nano-oscillators, ${ }^{63}$ and magnetic random-access memories. ${ }^{64}$ In the simplest case, a uniform current density may be used, with the LLG equation augmented with appropriate spin torque terms. More advanced solvers also allow for non-uniform current densities, thus enabling simulations of structures with non-constant cross-sectional area. In Boris, the current density may be computed self-consistently for any given geometry and multi-layered structure without having to import a computed current density, using the successive over-relaxation method. ${ }^{65}$

For charge transport only, this is given by $\mathbf{J}_{c}=\sigma \mathrm{E}$, where $\sigma$ is the electrical conductivity and $\mathbf{E}=-\nabla V$ is the electric field obtained from the electrical potential $V$. Results in Fig. 4 made use of the LLG-STT equation with a uniform current density. Here, we further show computations with non-uniform current densities and, in particular, we compute the AMR in a $\mathrm{Ni}_{80} \mathrm{Fe}_{20}$ ellipse where a current density is generated by applying a potential drop across two $5 \mathrm{~nm}$ thick metallic contacts, which are included as separate computational meshes with $\mathrm{Ru}$ material and Dirichlet boundary conditions set at the ends. The contacts are placed on top of the ellipse, with the simulated geometry given in Fig. 7(a) showing the computed current density. Composite media boundaries between the ellipse and the contacts are treated using the continuity of flux (current density) and electrical potential. The AMR effect is included as $\sigma=\sigma_{0} /\left(1+r d^{2}\right)$, where $\sigma_{0}$ is the material base conductivity, $r$ is the AMR ratio taken as 0.02 for $\mathrm{Ni}_{80} \mathrm{Fe}_{20}$, and $d=\mathbf{J}_{C} \cdot \mathbf{M} / \mid$ $\mathbf{J}_{C} \| \mathbf{M} \mid$. The results are shown in Fig. 7(b) where the resistance is obtained as the potential drop across the entire simulated structure divided by the total current flowing into the circuit ground. The AMR loops show the typical behavior expected for longitudinal and transverse AMR loops, and it is noteworthy the computed resistance change is significantly lower than the input AMR parameter. This is mostly due to the inclusion of constant resistance of the simulated electrical contacts, but also due to non-uniformity of the current density. Test simulations with uniform current density and potential drop applied directly across a single ferromagnetic mesh reproduce the input AMR ratio accurately. 

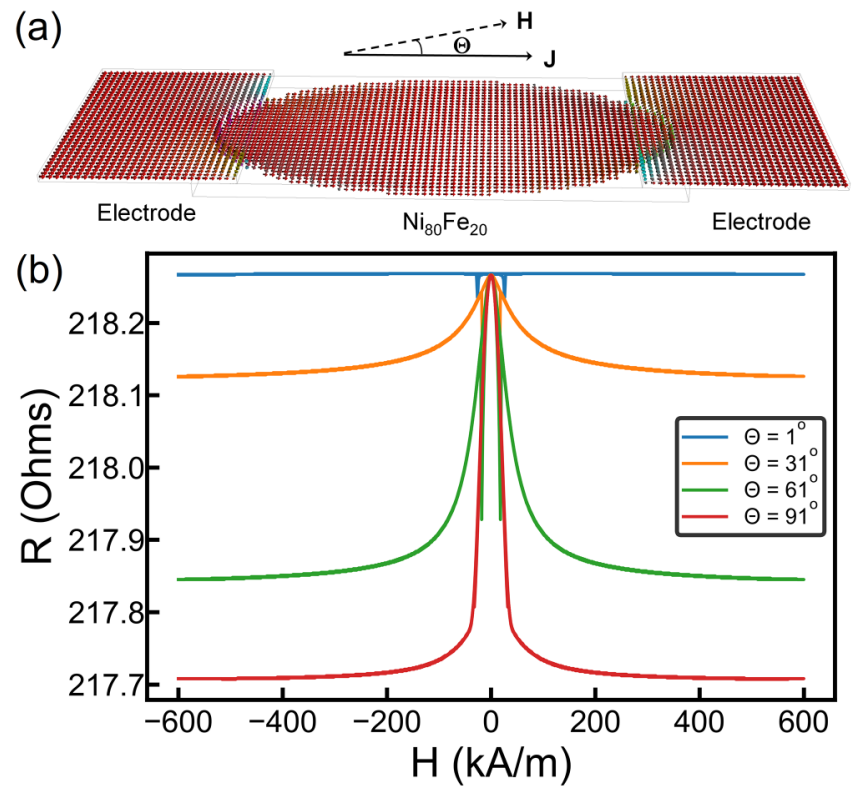

FIG. 7. Anisotropic magneto-resistance (AMR) in a $320 \times 160 \times 10 \mathrm{~nm}^{3} \mathrm{Ni}_{80} \mathrm{Fe}_{20}$ ellipse. (a) Simulation geometry showing the computed current density, and (b) AMR loops computed for different angles to the ellipse long axis.

An additional benefit of self-consistently computing current densities, an Oersted field can then be generated from it. This avoids having to compute the Oersted field externally and then importing it into the program, which apart from constant current densities is not trivial. Moreover, internal computation of the Oersted field allows simulations with the time-dependent Oersted field, for example, due to time-dependent current densities. Boris computes the Oersted field from the current density by evaluating the convolution sum with an Oersted tensor, using the formulas given in Ref. 66.

Additionally, Boris also allows computation of spin transport based on the drift-diffusion model, ${ }^{14,15}$ augmented with circuit theory boundary conditions. ${ }^{16}$ The full system of equations implemented is shown below,

$$
\begin{aligned}
\mathbf{J}_{C}= & \sigma \mathbf{E}+\beta_{D} D_{e} \frac{e}{\mu_{B}}(\nabla \mathbf{S}) \mathbf{m}+\theta_{S H A} D_{e} \frac{e}{\mu_{B}} \nabla \times \mathbf{S}+P \sigma \frac{\hbar}{2 e} \mathbf{E}^{\sigma}-P \frac{\sigma^{2} \hbar}{e^{2} n} \mathbf{E} \times \mathbf{B}^{\sigma}, \\
\mathbf{J}_{S}= & -\frac{\mu_{B}}{e} P \sigma \mathbf{E} \otimes \mathbf{m}-D_{e} \nabla \mathbf{S}+\theta_{S H A} \frac{\mu_{B}}{e} \boldsymbol{\varepsilon} \sigma \mathbf{E} \\
& +\frac{\hbar \mu_{B} \sigma}{2 e^{2}} \sum_{i} \mathbf{e}_{i} \otimes\left(\dot{\mathbf{m}} \times \partial_{i} \mathbf{m}\right)+\frac{\hbar \mu_{B} \sigma^{2}}{e^{3} n}(\mathbf{z} \times \mathbf{E}) \otimes\left(\partial_{x} \mathbf{m} \times \partial_{y} \mathbf{m}\right) .
\end{aligned}
$$

The charge current density now additionally includes contributions due to (i) current perpendicular to plane giant magnetoresistance (CPP-GMR), where $\beta_{\mathrm{D}}$ is the diffusion spin polarization, $D_{e}$ is the electron diffusion constant, and $\mathbf{S}$ is the spin accumulation. (ii) ISHE where $\theta_{S H A}$ is the intrinsic spin Hall angle, (iii) charge pumping, and (iv) topological Hall effect, where $E_{i}^{\sigma}=$ $\left(\dot{\mathbf{m}} \times \partial_{i} \mathbf{m}\right) \cdot \mathbf{m}$ and $\mathbf{B}^{\sigma}=\mathbf{z}\left(\partial_{x} \mathbf{m} \times \partial_{y} \mathbf{m}\right) \cdot \mathbf{m}$. Here, $\mathbf{E}^{\sigma}$ and $\mathbf{B}^{\sigma}$ are the directions of the emergent electric field due to charge pumping and emergent magnetic field due to the topological Hall effect, respectively. ${ }^{20,21}$ The spin current density tensor, where $\mathbf{J}_{S i j}$ indicates the flow of the $j$ component of spin polarization in the direction $i$, includes contributions due to (i) drift, (ii) diffusion, (iii) SHE, where $\epsilon$ is the rank 3 unit antisymmetric tensor, (iv) charge pumping, and (v) the topological Hall effect where $n$ is the itinerant electron density. The spin accumulation obeys the following equation of motion, where $\lambda_{s f}$ is the spin flip length, $\lambda_{J}$ is the exchange rotation length, and $\lambda_{\varphi}$ is the spin dephasing length:

$$
\frac{\partial \mathbf{S}}{\partial t}=-\nabla \cdot \mathbf{J}_{S}-D_{e}\left(\frac{\mathbf{S}}{\lambda_{s f}^{2}}+\frac{\mathbf{S} \times \mathbf{m}}{\lambda_{J}^{2}}+\frac{\mathbf{m} \times(\mathbf{S} \times \mathbf{m})}{\lambda_{\phi}^{2}}\right) .
$$

Solving for the spin accumulation allows computation of bulk spin torques, which may be included as an additional torque term in the LLG equation, as

$$
\mathbf{T}_{S}=-\frac{D_{e}}{\lambda_{J}^{2}} \mathbf{m} \times \mathbf{S}-\frac{D_{e}}{\lambda_{\phi}^{2}} \mathbf{m} \times(\mathbf{m} \times \mathbf{S}) .
$$

It may be shown that under the assumption of negligible in-plane spin diffusion this expression is equivalent to Zhang-Li STTs as given in Eq. (2), 9,17,67 where the non-adiabaticity parameter is constant and given by $\beta \cong \lambda_{J}^{2} / \lambda_{s f}^{2}$ in the limit of long spin dephasing length and long domain walls. The assumption of negligible in-plane spin diffusion breaks down for rapidly varying magnetization textures such as vortices and skyrmions, and this can lead to spatially varying and enhanced non-adiabaticity. For example, it is known that vortex domain walls have a significantly larger non-adiabaticity compared to transverse domain walls, ${ }^{68}$ arising mainly due to in-plane spin diffusion at large magnetization gradients, with contributions due to charge pumping and the topological Hall effect also recognized. ${ }^{69}$ While it may still be possible to use the simple LLG-STT formulation of Eq. (2), the correct value of non-adiabaticity must be used when vortex domain walls are present, and this may be computed using the drift-diffusion model. This is shown in Fig. 4(a), where for $\mathrm{Ni}_{80} \mathrm{Fe}_{20}$ the relation $\beta \cong \lambda_{J}^{2} / \lambda_{s f}^{2}$ gives $\beta=0.04$ expected for a transverse domain wall with $\lambda_{s f}=10 \mathrm{~nm}$ and $\lambda_{J}=2 \mathrm{~nm}$. For the vortex domain wall in Fig. 4(a) however a much higher maximum value of 0.1 results for $\lambda_{\varphi}=2.1 \mathrm{~nm}$, obtained by fitting the spin torque in Eq. (8) to the STT in Eq. (2) with $\beta$ as a spatially varying fitting parameter. Thus, $\beta$ is no longer a constant but has a spatial dependence with the maximum value reached at the vortex core as seen in Fig. 4(a). The $\mu$ MAG Standard Problem \#5 is repeated again, but this time the LLG equation is used with the spin torque from Eq. (8), i.e., the spin accumulation is solved at every time step to self-consistently compute the spin torque. The results are shown in Fig. 4(b) where a good agreement is obtained with the LLG-STT equation, despite the very different methods used to solve the problem.

At non-magnetic ( $\mathrm{N})$ /ferromagnetic $(\mathrm{F})$ composite media boundaries, the following conditions are applied, obtained from circuit theory using the spin mixing conductance $G^{\uparrow \downarrow}$ and interface conductances for majority and minority carriers, $G^{\uparrow}$ and $G^{\downarrow}$ : 


$$
\begin{aligned}
& \left.\mathbf{J}_{C} \cdot \mathbf{n}\right|_{N}=\left.\mathbf{J}_{C} \cdot \mathbf{n}\right|_{F}=-\left(G^{\uparrow}+G^{\downarrow}\right) \Delta V+\left(G^{\uparrow}-G^{\downarrow}\right) \Delta \mathbf{V}_{S} \cdot \mathbf{m}, \\
& \left.\mathbf{J}_{S} \cdot \mathbf{n}\right|_{N}-\left.\mathbf{J}_{S} \cdot \mathbf{n}\right|_{F}=\frac{2 \mu_{B}}{e}\left[\operatorname{Re}\left\{G^{\uparrow \downarrow}\right\} \mathbf{m} \times\left(\mathbf{m} \times \Delta \mathbf{V}_{S}\right)+\operatorname{Im}\left\{G^{\uparrow \downarrow}\right\} \mathbf{m} \times \Delta \mathbf{V}_{S}\right], \\
& \left.\mathbf{J}_{S} \cdot \mathbf{n}\right|_{F}=\frac{\mu_{B}}{e}\left[-\left(G^{\uparrow}+G^{\downarrow}\right)\left(\Delta \mathbf{V}_{S} \cdot \mathbf{m}\right) \mathbf{m}+\left(G^{\uparrow}-G^{\downarrow}\right) \Delta V \mathbf{m}\right] .
\end{aligned}
$$

Interfacial spin torques are obtained as $\left(h_{F}\right.$ is the discretization cellsize of the $F$ layer in the direction normal to the composite media boundary)

$$
\mathbf{T}_{S}=\frac{g \mu_{B}}{e h_{F}}\left[\operatorname{Re}\left\{G^{\uparrow \downarrow}\right\} \mathbf{m} \times\left(\mathbf{m} \times \Delta \mathbf{V}_{S}\right)+\operatorname{Im}\left\{G^{\uparrow \downarrow}\right\} \mathbf{m} \times \Delta \mathbf{V}_{S}\right] .
$$

Spin pumping may also be included on the $\mathrm{N}$ side of Eq. (9) as

$$
\mathbf{J}_{S}^{p u m p}=\frac{\mu_{B} \hbar}{e^{2}}\left[\operatorname{Re}\left\{G^{\uparrow \downarrow}\right\} \mathbf{m} \times \frac{\partial \mathbf{m}}{\partial t}+\operatorname{Im}\left\{G^{\uparrow \downarrow}\right\} \frac{\partial \mathbf{m}}{\partial t}\right] .
$$

This results in a damping-like torque in Eq. (10), reproducing the expected enhancement in effective magnetization damping. ${ }^{17}$ As shown previously, when a heavy metal (HM)/F bilayer is simulated with the SHE enabled in the HM layer, the expected damping-like and field-like SOTs are obtained from Eq. (10). ${ }^{17,70}$ Moreover, when a spin accumulation is generated at magnetization

(a)

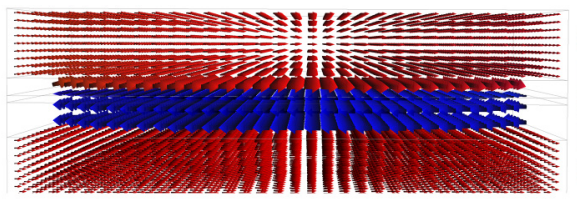

Contact Free Laver Spacer Fixed Layer Contact

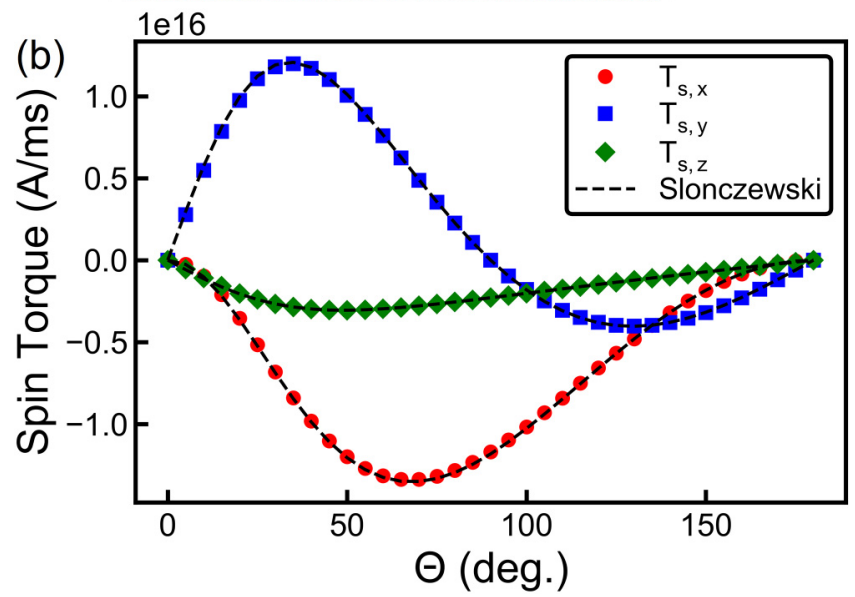

FIG. 8. Spin torques in a CPP-GMR spin valve structure for a current density of $\sim 10^{12} \mathrm{~A} / \mathrm{m}^{2}$. (a) Spin-valve geometry with dimensions $160 \times 80 \mathrm{~nm}^{2}, 10 \mathrm{~nm}$ fixed layer thickness, $5 \mathrm{~nm}$ free layer thickness, $2 \mathrm{~nm}$ spacer thickness, and contacts of $20 \mathrm{~nm}$ thickness. (b) Computed spin torques in the free layer for uniform magnetization as a function of in-plane angle, with fitted Slonczewski and fieldlike spin torques. gradients, such as a skyrmion, the resulting imbalance in spin accumulation either side of the HM/FM interface generates vertical spin currents, which leads to an additional type of interfacial spin torque, termed interfacial STT (ISTT).$^{10,11}$ In many cases, it is sufficient to run simulations with direct expressions for spin torques (e.g., STT and SOT) augmenting the LLG equation; however, the drift-diffusion spin transport solver is still useful for calculating the strength of these spin torques in the first place from spin transport parameters. Here, we further verify the spin transport solver reproduces the expected spin torques in a spin valve structure shown in Fig. 8(a). In a macrospin approximation, the total spin torque exerted on the free layer is given by a combination of Slonczewski and field-like spin torques as ${ }^{71,72}$

$$
\begin{aligned}
& \mathbf{T}_{S}=\frac{\mu_{B}}{e} \frac{J}{d} \eta(\theta)[\mathbf{m} \times(\mathbf{m} \times \mathbf{p})+r \mathbf{m} \times \mathbf{p}], \\
& \eta(\theta)=\frac{q_{+}}{A+B \cos (\theta)}+\frac{q_{-}}{A-B \cos (\theta)} .
\end{aligned}
$$

Here, $\mathbf{p}$ is the polarization from the fixed layer, set to $\mathbf{p}=-\hat{\mathbf{x}}$. By varying the angle in the uniformly magnetized free layer, the angular dependence $\eta(\theta)$ in Eq. (12) is obtained by fitting the spin torque computed self-consistently in Eq. (10). The results are shown in Fig. 8(b), where we obtain $q_{+}=4.94, q_{-}=-0.05, A=5.85$, $B=3.83$, and $r=0.19$. It should be noted that while a good agreement is obtained between Eqs. (10) and (12) for the angular dependence, the model above is strictly applicable for a macrospin only. During switching of the free layer, the magnetization is no longer uniform resulting in non-negligible spin diffusion effects. While the switching times computed with Eqs. (10) and (12), respectively, are approximately the same for the geometry in Fig. 8(a), the exact magnetization dynamics of the free layer differ between them. In this case, the self-consistent spin torque in Eq. (10) is a more accurate description, capturing the non-local nature of the spin torques.

\section{LANDAU-LIFSHITZ-BLOCH EQUATION}

Material parameters used in simulations may be assigned temperature dependences, either using a data file or with a usersupplied mathematical equation. This is particularly useful for computations where the temperature can change during the simulation. In this case, the (stochastic) Landau-Lifshitz-Bloch (LLB) equation ${ }^{8}$ is used, which is given by

$$
\begin{aligned}
\frac{\partial \mathbf{M}}{\partial t}= & -\frac{\gamma}{1+\tilde{\alpha}_{\perp}^{2}} \mathbf{M} \times \mathbf{H}-\frac{\tilde{\alpha}_{\perp} \gamma}{1+\tilde{\alpha}_{\perp}^{2}} \frac{1}{|\mathbf{M}|} \mathbf{M} \\
& \times\left(\mathbf{M} \times\left(\mathbf{H}+\mathbf{H}_{\text {thermal }}\right)\right)+\frac{\gamma \tilde{\alpha}_{\|}}{|\mathbf{M}|}(\mathbf{M} . \mathbf{H}) \mathbf{M}+\boldsymbol{\eta}_{\text {thermal }} .
\end{aligned}
$$


Here, for $T<T_{C} \quad\left(T_{C} \quad\right.$ is the Curie temperature), $\alpha_{\perp}=\alpha\left(1-T / 3 T_{C}\right), \alpha_{\|}=\alpha 2 T / 3 T_{C}, \tilde{\alpha}_{\perp}=\alpha_{\perp} / m$, and $\tilde{\alpha}_{\|}=\alpha_{\|} / m$, where $m$ is the magnetization length normalized to its zero temperature value, i.e., $m=|\mathbf{M}| / M_{S}^{0}$. For $T>T_{C}, \alpha_{\perp}=\alpha_{\|}=2 T / 3 T_{C}$. The effective field $\mathbf{H}$ must be complemented by a longitudinal susceptibility field given by

$$
\mathbf{H}_{l}= \begin{cases}\left(1-\frac{m^{2}}{m_{e}^{2}}\right) \frac{\mathbf{m}}{2 \mu_{0} \tilde{\chi}_{\|}}, & T \leq T_{C}, \\ -\frac{\mathbf{m}}{\mu_{0} \tilde{\chi}_{\|}}, & T>T_{C} .\end{cases}
$$

The field and temperature-dependent equilibrium magnetization, $m_{e}$, is obtained from the Curie-Weiss law as

$$
m_{e}(T)=B\left[m_{e} \frac{3 T_{C}}{T}+\frac{\mu \mu_{0} H_{e x t}}{k_{B} T}\right],
$$

where $B(x)=\operatorname{coth}(x)-1 / x$ is the Langevin function and $\mu$ is the atomic moment. The longitudinal susceptibility is $\chi_{\|}(T)=\partial M /\left.\partial H\right|_{H \rightarrow 0},{ }^{6,8}$ and from this we obtain the relative longitudinal susceptibility (units $\mathrm{T}^{-1}$ )

$$
\begin{aligned}
\tilde{\chi}_{\|}(T) & =\frac{\mu}{k_{B} T} \frac{B^{\prime}(x)}{1-B^{\prime}(x)\left(3 T_{C} / T\right)}=\chi_{\|}(T) / \mu_{0} M_{S}^{0}, \quad \text { with } \\
x & =m_{e} 3 T_{C} / T .
\end{aligned}
$$

The components of the thermal field, $\mathbf{H}_{\text {thermal }}$, and torque, $\boldsymbol{\eta}_{\text {thermal }}$, follow Gaussian distributions with no correlations, zero mean, and standard deviations given, respectively, by

$$
\begin{aligned}
& H_{\sigma}=\frac{1}{\alpha_{\perp}} \sqrt{\frac{2 k_{B} T\left(\alpha_{\perp}-\alpha_{\|}\right)}{\gamma \mu_{0} M_{S}^{0} V \Delta t}}, \\
& \eta_{\sigma}=\sqrt{\frac{2 k_{B} T \alpha_{\|} \gamma M_{s}^{0}}{\mu_{0} V \Delta t}} .
\end{aligned}
$$

Here, $V$ is the computational cellsize and $\Delta t$ is the integration time step. Stochastic equations are evaluated in Boris in the Stratonovich interpretation, ${ }^{73}$ and applicable methods include the Euler and Heun methods (both fixed and adaptive time step)-the other previously mentioned higher order methods are not suitable for stochastic equations.

Temperature dependences of parameters may be adjusted by the user as explained above; however, when a Curie temperature is set the program computes a set of default temperature dependences, which are given as scaling laws on the base (zero-temperature) material parameter values. These are $M_{S}(T)=M_{S}^{0} m_{e}(T)$ and $A(T)=$ $A_{0} m_{e}^{2}(T)$ for the exchange stiffness, ${ }^{74} D(T)=D_{0} m_{e}^{2}(T)$ for the Dzyaloshinsky-Moriya exchange constant, ${ }^{75}$ and $K(T)=K_{0} m_{e}^{3}(T)$ for the anisotropy constants. ${ }^{7,77}$ It must be noted, however, the default temperature dependences are not always appropriate and may need to be adjusted depending on the material simulated, for example, see Refs. 78 and 79. For the full list of effective fields implemented, see Appendix A.

\section{HEAT SOLVER}

In the simplest case, the electron temperature in the LLB equation is uniform. More advanced simulations also require non-uniform temperatures, and this calls for implementation of the heat equation, although an externally computed temperature distribution can also be loaded into Boris. For example, the authors in Ref. 22 investigated the AMR-induced magnonic spin-Seebeck effect, where Joule heating is included in the heat equation as the heat source $\mathrm{J}^{2} / \sigma\left(\mathrm{W} / \mathrm{m}^{3}\right)$. Due to the AMR of a transverse $\mathrm{DW}$, the conductivity is higher at the DW, locally resulting in decreased Joule heating. This results in a temperature gradient between the center of the DW and its boundaries, and moreover when the DW is displaced due to STT the leading edge of the DW experiences a higher temperature compared to the trailing edge. Due to the magnonic spin-Seebeck effect, ${ }^{80}$ this results in a significant enhancement of the DW velocity up to $15 \%$ for realistic material parameters. ${ }^{22}$ To reproduce such an effect, it is necessary to simultaneously solve both the LLB and heat equations, in addition to computing the current density using the transport solver. The heat equation implemented in Boris is shown below,

$$
C(\mathbf{r}) \rho(\mathbf{r}) \frac{\partial T(\mathbf{r}, t)}{\partial t}=\nabla \cdot K(\mathbf{r}) \nabla T(\mathbf{r}, t)+S(\mathbf{r}, t)+\frac{\mathbf{J}(\mathbf{r}, t)^{2}}{\sigma(\mathbf{r})} .
$$

Here, $C$ is the total specific heat capacity, $K$ is the thermal conductivity, $\rho$ is the mass density, and $S$ is the heat source. The material parameters in the heat equation are allowed to vary spatially, for example, if a temperature dependence is enabled. All material parameters, including the heat source $S$, in Boris may be assigned a temperature dependence, specified either through a data file or through a user-defined mathematical equation. The final term in Eq. (18) represents Joule heating, and the heat solver may be coupled to the transport solver as explained in relation to the example in Ref. 22 . The heat equation is evaluated using the forward-time centered-space method. While this is a first-order method, so the time step required for stability is small, this is in many cases comparable to the time step required for evaluating the LLB equation; thus, a higher order scheme is not necessary.

For more advanced studies, which require separating the lattice and electron temperatures, a two-temperature model ${ }^{23}$ is available, which is given as

$$
\begin{aligned}
& C_{e}(\mathbf{r}) \rho(\mathbf{r}) \frac{\partial T_{e}(\mathbf{r}, t)}{\partial t}=\nabla \cdot K(\mathbf{r}) \nabla T_{e}(\mathbf{r}, t)-G_{e}(\mathbf{r})\left[T_{e}(\mathbf{r}, t)-T_{l}(\mathbf{r}, t)\right]+S(\mathbf{r}, t) \\
& C_{l}(\mathbf{r}) \rho(\mathbf{r}) \frac{\partial T_{l}(\mathbf{r}, t)}{\partial t}=G_{e}(\mathbf{r})\left[T_{e}(\mathbf{r}, t)-T_{l}(\mathbf{r}, t)\right] .
\end{aligned}
$$

Here, $C_{e}$ and $C_{l}$ are the electron and lattice specific heat capacities, and $G_{e}$ is the electron-lattice coupling constant, typically of the order $10^{18} \mathrm{~W} / \mathrm{m}^{3} \mathrm{~K}$. When used together with the LLB equation, the magnetization and magnetic parameters are coupled to the electron temperature $\left(T=T_{e}\right)$. 
Studies of ultrafast magnetization dynamics have revealed a large difference between electron and spin dynamics on time scales of the order 1 ps and below, explained in terms of a three-temperature model which includes the electron, spin, and lattice temperatures, ${ }^{81}$ and later formulated as a microscopic three-temperature model. ${ }^{82}$ This latter approach was shown to be equivalent to an LLB formulation, ${ }^{23}$ which accounts for the different electron and lattice temperatures on ultra-short time scales. Within this formulation, the photon energy is absorbed by the delocalized electrons, which are coupled to the lattice electrons via the rate equations shown in Eq. (19). This allows simulations of ultrafast demagnetization due to heating by a laser pulse, by including an appropriate heat source $S$ in Eq. (19). Here, we show an example of such a simulation by taking a Gaussian profile for a linearly polarized laser pulse as given below, where $d$ and $t_{R}$ are full-width at half-maximum values for the spatial and temporal widths,

$$
S=P_{0} \exp \left(\frac{-\left|\mathbf{r}-\mathbf{r}_{0}\right|}{d^{2} / 4 \ln (2)}\right) \exp \left(\frac{-\left(t-t_{0}\right)^{2}}{t_{R}^{2} / 4 \ln (2)}\right) \quad\left(\mathrm{W} / \mathrm{m}^{3}\right)
$$

The geometry simulated is shown in Fig. 9(a), where we use a $\mathrm{Co}(2 \mathrm{~nm}) / \mathrm{Pt}(8 \mathrm{~nm}) / \mathrm{SiO}_{2}(40 \mathrm{~nm})$ structure, with in-plane dimensions of $512 \mathrm{~nm}^{2}$ and periodic boundary conditions. For the Co layer, we also include the interfacial DMI contribution and

(a)

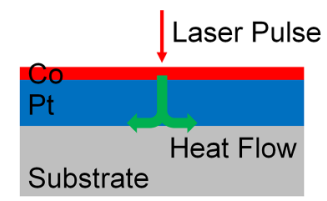

(b)
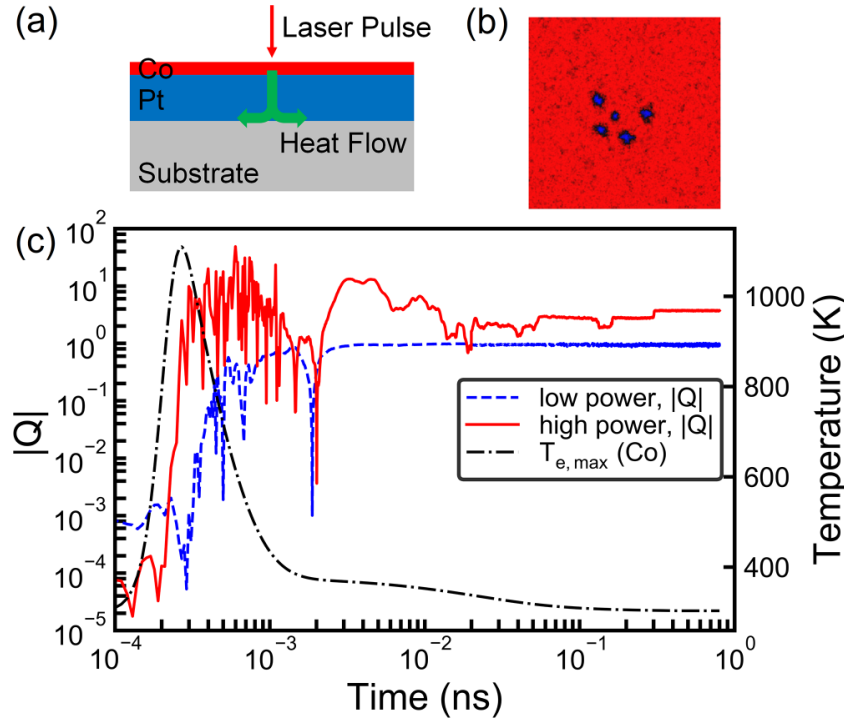

FIG. 9. Ultrafast demagnetization and Néel skyrmion creation in a $2 \mathrm{~nm}$ thick Co layer on $\mathrm{Pt}(8 \mathrm{~nm})$ and $\mathrm{SiO}_{2}$ substrate $(40 \mathrm{~nm})$. (a) Simulated trilayer structure. (b) State after $800 \mathrm{ps}$ for a high power laser pulse $\left(T_{\max } \cong 2 T_{C}\right.$, $\left.T_{C}=500 \mathrm{~K}\right)$ and out-of-plane field of $100 \mathrm{kA} / \mathrm{m}$, showing the $z$ component of magnetization and five created skyrmions. (c) $|Q|$ plotted as a function of time for two different pulse strengths (high $T_{\max } \cong 2 T_{C}$, low $T_{\max } \cong 1.5 T_{C}$ ) and $d=400 \mathrm{~nm}, t_{R}=100 \mathrm{fs}$. The maximum Co temperature reached for the high power laser pulse is also plotted. uniaxial anisotropy with easy axis out of the plane. The twotemperature model is computed for the Co and Pt layers, while the one-temperature heat equation is computed for the $\mathrm{SiO}_{2}$ substrate. Continuity of heat flux and temperature is assumed at the interfaces, and Robin boundary conditions are used on the exposed surfaces of the magnetic layer and substrate with ambient temperature set to room temperature. For a high power laser pulse $\left(P_{0}=4 \times 10^{21} \mathrm{~W} / \mathrm{m}^{3}\right)$ with $d=400 \mathrm{~nm}$ and $t_{R}=100 \mathrm{fs}$, the computed maximum Co electron temperature is plotted in Fig. 9(c), showing ultrafast heating up to $T_{\max } \cong 2 T_{C}\left(T_{C}=500 \mathrm{~K}\right)$, followed by rapid cooling as the electron and lattice temperatures equilibrate. The temperature decays back to room temperature on a longer time scale. For this problem, we solve the stochastic LLB in Eq. (13) and compute the topological charge (which takes on values \pm 1 for a single skyrmion) using ${ }^{83}$

$$
Q=\frac{1}{4 \pi} \int_{A} \mathbf{m} \cdot\left(\frac{\partial \mathbf{m}}{\partial x} \times \frac{\partial \mathbf{m}}{\partial y}\right) d x d y
$$

Thus, by plotting $|\mathrm{Q}|$ as a function of time, the number of skyrmions present can be monitored. As the magnetization order recovers for $T<T_{C}$ following ultrafast demagnetization, Néel skyrmions begin to emerge under the action of DMI, as observed experimentally. ${ }^{84}$ The mean number of skyrmions formed is dependent on the laser power and follows a Poisson counting distribution as discussed in Ref. 85. Two examples are shown in Fig. 9(c): the low power pulse results in a single skyrmion formed in this case, while the high power pulse results in five skyrmions formed, with the final state indicated in Fig. 9(b). The integration of a multi-layered heat solver with the magnetization dynamics solver is thus a powerful feature, allowing detailed studies with non-uniform and non-constant temperatures and heat sources.

\section{TWO-SUBLATTICE MODEL}

Recent years have seen an increased interest in antiferromagnetic spintronics, ${ }^{86-88}$ with the real prospect of antiferromagnetic memories ${ }^{89}$ in sight, and applications to terahertz technologies. ${ }^{90}$ Thus, micromagnetics research software is needed to support future efforts in this area. Following the multi-mesh and multimaterial paradigm, Boris has been extended with a two-sublattice model, allowing modeling of antiferromagnetic and ferrimagnetic materials, for example, applicable to studies of ferrimagnetic skyrmions. ${ }^{91}$ This allows studying not only antiferromagnetic and ferrimagnetic materials devices on their own, but also complex multi-layered devices including both antiferromagnetic and ferromagnetic materials-one obvious application here is to the study of exchange bias. $^{92}$

Here, we show the two-sublattice stochastic LLB equation implemented in Boris, based on the LLB equation from Refs. 93 and 94 applicable for antiferromagnetic, ferrimagnetic, and binary ferromagnetic alloys. This is given in Eq. (22) in terms of the macroscopic magnetization, where we denote the two sublattices as $i=A, B$, 


$$
\begin{aligned}
\frac{\partial \mathbf{M}_{i}}{\partial t}= & -\tilde{\gamma}_{i} \mathbf{M}_{i} \times \mathbf{H}_{e f f, i}-\tilde{\gamma}_{i} \frac{\tilde{\alpha}_{\perp, i}}{M_{i}} \mathbf{M}_{i} \times\left(\mathbf{M}_{i} \times\left(\mathbf{H}_{e f f, i}+\mathbf{H}_{t h, i}\right)\right) \\
& +\gamma_{i} \frac{\tilde{\alpha}_{\|, i}}{M_{i}}\left(\mathbf{M}_{i} \cdot \mathbf{H}_{\|, i}\right) \mathbf{M}_{i}+\mathbf{\eta}_{t h, i} \quad(i=A, B) .
\end{aligned}
$$

The reduced gyromagnetic ratio is given by $\tilde{\gamma}_{i}=\gamma_{i} /\left(1+\tilde{\alpha}_{\perp, i}^{2}\right)$, and the reduced transverse and longitudinal damping parameters by $\tilde{\alpha}_{\perp(||), i}=\alpha_{\perp(\|), i} / m_{i}$, where $m_{i}(T)=M_{i}(T) / M_{S, i}^{0}$, with $M_{S, i}^{0}$ denoting the zero-temperature saturation magnetization and $M_{i} \equiv\left|\mathbf{M}_{\mathrm{i}}\right|$. The exchange field now includes not only intra-lattice contributions, but also homogeneous and non-homogeneous interlattice contributions given as

$$
\begin{aligned}
\mathbf{H}_{e x, i} & =\frac{2 A_{i}}{\mu_{0} M_{e, i}^{2}} \nabla^{2} \mathbf{M}_{i}-\frac{4 A_{h, i}}{\mu_{0} M_{e, i} M_{e, j}} \hat{\mathbf{m}}_{i} \times\left(\hat{\mathbf{m}}_{i} \times \mathbf{M}_{j}\right)+\frac{A_{n h, i}}{\mu_{0} M_{e, i} M_{e, j}} \nabla^{2} \mathbf{M}_{j} \\
(i=A, B, i \neq j) . &
\end{aligned}
$$

The effective field includes a number of contributions, as for the ferromagnetic model, namely, demagnetizing field computed for $\left(\mathbf{M}_{A}+\mathbf{M}_{B}\right) / 2$, external field, magneto-crystalline anisotropy, as well as DMI or interfacial DMI terms. The formulas given in Appendix A are now applicable to the two sublattices separately.

The relative longitudinal susceptibility, $\tilde{\chi}_{\|, i}=\chi_{\|, i} / \mu_{0} M_{S, i}^{0}$, now becomes ${ }^{94}$

$k_{B} T \tilde{\chi}_{\|, i}=\frac{\mu_{i} B_{i}^{\prime}\left(1-3 \tau_{j} \tilde{T}_{N} B^{\prime}{ }_{j} / T\right)+\mu_{j} 3 \tau_{i j} \tilde{T}_{N} B_{i}^{\prime} B_{j}^{\prime} / T}{\left(1-3 \tau_{i} \tilde{T}_{N} B_{i}^{\prime} / T\right)\left(1-3 \tau_{j} \tilde{T}_{N} B^{\prime}{ }_{j} / T\right)-\tau_{i j} \tau_{j i} B_{i}^{\prime} B_{j}^{\prime}\left(3 \tilde{T}_{N} / T\right)^{2}}$,

where $B_{i}^{\prime} \equiv B_{m_{e, i}}^{\prime}\left[\left(m_{e, i} \tau_{i}+m_{e, j} \tau_{i j}\right) 3 \tilde{T}_{N} / T\right](i=A, B)$. Here, $m_{e, i}$ are magnetization temperature scaling laws, and $\tau_{i}, \tau_{i j}$ are dimensionless coupling parameters between the effective exchange parameters and the phase transition temperature, which allow convenient specification of materials for two-sublattice micromagnetic simulations. Full details, including the longitudinal relaxation field expression, and examples are given in Appendix B.

Here, we test the two-sublattice model by computing the antiferromagnetic resonance (AFMR) as a function of antiferromagnetic exchange and uniaxial anisotropy. The predicted resonance frequency is given by the Kittel formula ${ }^{95}$ as $f_{0}=\sqrt{H_{A}\left(2 H_{E}+H_{A}\right)}$ at zero external field, where $H_{A}=2 K_{1} / \mu_{0} M_{S}$, and $H_{E}=4\left|A_{h}\right| / \mu_{0} M_{s}$. We compute the resonance frequency for a generic antiferromagnetic material with $M_{S}=800 \mathrm{kA} / \mathrm{m}$ and $A=13 \mathrm{pJ} / \mathrm{m}$ on each sublattice, as a function of homogeneous antiferromagnetic exchange and uniaxial anisotropy constant, by applying a uniform sinc pulse and taking the Fourier transform to obtain a frequency-swept AFMR peak. The excitation is applied perpendicular to the easy axis with amplitude $1 \mathrm{kA} / \mathrm{m}$. Results are plotted in Fig. 10(a), showing a good agreement with the Kittel formula over a wide range of values. We further compute the spin wave dispersion with the same method used to compute the ferromagnetic spin wave dispersion in Fig. 3. Here, we set $A=5 \mathrm{pJ} / \mathrm{m}, A h=-20 \mathrm{MJ} / \mathrm{m}^{3}, K_{1}=50 \mathrm{~kJ} / \mathrm{m}^{3}$ and also set a nonhomogeneous exchange constant $A_{n h}=-10 \mathrm{pJ} / \mathrm{m}$, with a damping constant of 0.002 . The $n=0$ spin wave mode analytical formula is given as

$$
w_{0}(k)=\gamma \sqrt{\left(2 H_{E}+H_{A}+\left(\frac{2 A+\left|A_{n h}\right|}{\mu_{0} M_{S}}\right) k^{2}\right)\left(H_{A}+\left(\frac{2 A+\left|A_{n h}\right|}{\mu_{0} M_{S}}\right) k^{2}\right)} .
$$

The results are plotted in Fig. 10(b), showing an excellent agreement with Eq. (25).

The two-sublattice model in Eq. (22) also includes stochastic terms, which is similar to the stochastic LLB equation having zero spatial, vector components, and inter-lattice correlations, and whose components follow Gaussian distributions with zero mean and standard deviations given by

$$
\begin{aligned}
H_{t h, i}^{s t d .} & =\frac{1}{\alpha_{\perp, i}} \sqrt{\frac{2 k_{B} T\left(\alpha_{\perp, i}-\alpha_{\|, i}\right)}{\gamma_{i} \mu_{0} M_{S, i}^{0} V \Delta t}} \\
\eta_{t h, i}^{s t d .} & =\sqrt{\frac{2 k_{B} T \alpha_{\|, i} \gamma_{i} M_{S, i}^{0}}{\mu_{0} V \Delta t}} .
\end{aligned}
$$

Similarly to the approach in Ref. 8, it can be shown that the magnetization length distribution follows a Boltzmann probability distribution. For the two-sublattice case, in general, this distribution is a function of the magnetization length of both sublattices, $m_{A}$ and $m_{B}$, and is shown below for the isotropic case (see Appendix B for further definitions),

$$
P_{i}\left(m_{A}, m_{B}\right) \propto m_{i}^{2} \exp \left\{-\frac{M_{S, i}^{0} V}{4 \mu_{i} m_{e, i} k_{B} T}\left[\frac{\left(m_{i}^{2}-m_{e, i}^{2}\right)^{2}}{m_{e, i}} \frac{\left(\mu_{i}+3 \tau_{i j} k_{B} T_{N} \tilde{\chi}_{\|, j}\right)}{2 \tilde{\chi}_{\|, i}}+\frac{\left(m_{j}^{2}-m_{e, j}^{2}\right)}{m_{e, j}} 3 \tau_{i j} k_{B} T_{N} m_{i}^{2}\right]\right\}(i, j=A, B, i \neq j)
$$

We test this by computing a two-sublattice histogram for the magnetization length as a function of temperature, taking the generic antiferromagnetic material of Fig. 10(a) with a Néel temperature $T_{N}=500 \mathrm{~K}$. A temperature is set and a cubic block 

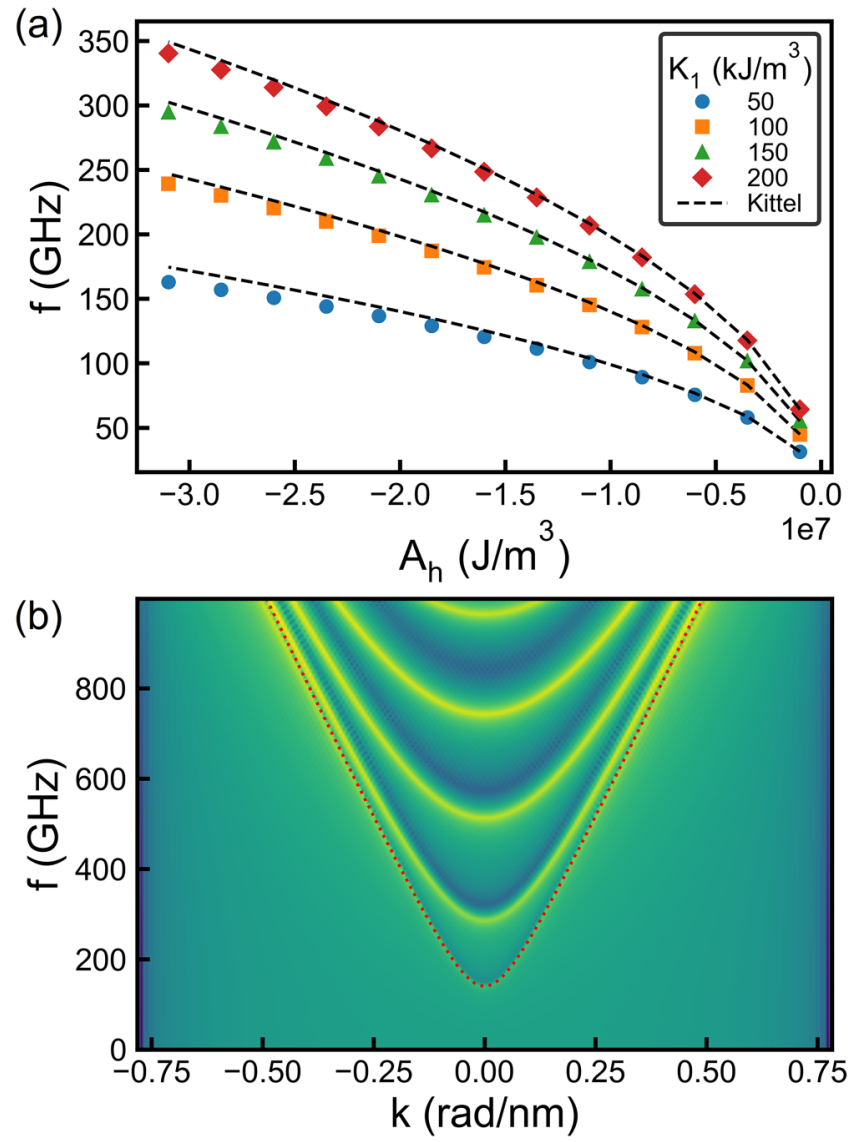

FIG. 10. (a) Antiferromagnetic resonance computed as a function of homogeneous antiferromagnetic exchange and uniaxial anisotropy, compared to the Kittel formula. (b) Antiferromagnetic spin wave dispersion computed for the same geometry in Ref. 38 with $A=5 \mathrm{pJ} / \mathrm{m}, A_{h}=-20 \mathrm{MJ} / \mathrm{m}^{3}, A_{n h}=-10 \mathrm{pJ} / \mathrm{m}$, and $K_{1}=50 \mathrm{~kJ} / \mathrm{m}^{3}$, using a damping factor of 0.002 , compared to Eq. (25) for $n=0$ mode (dotted line).

of antiferromagnetic material ( $400 \mathrm{~nm}$ side) with periodic boundary conditions in all directions is allowed to relax for a set time (20 ps or longer). The computed two-sublattice probability distribution is shown in Fig. 11 for $T / T_{N}=0.99$ as a color map. A very good agreement is obtained with the two-sublattice Boltzmann distribution from Eq. (27), plotted as a wire-frame. Similar tests were repeated over a wide range of temperatures. This shows that the implemented stochastic two-sublattice LLB model correctly reproduces the expected stochastic properties.

Exchange bias may also be modeled by simulating a bilayer consisting of an antiferromagnetic and a ferromagnetic mesh. The effective exchange bias field, first observed by Meiklejohn and Bean, ${ }^{96}$ coincides with the bilinear surface exchange field of Eq. (5); thus, exchange bias may be included by enabling the surface exchange fields at the interface between antiferromagnetic and ferromagnetic meshes. ${ }^{97}$ By including

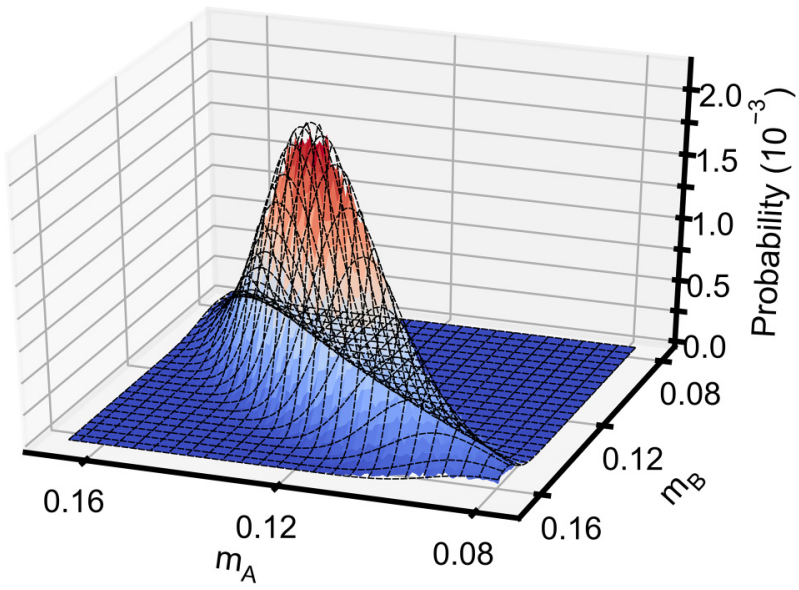

FIG. 11. Two-sublattice antiferromagnetic magnetization length probability distribution at $T / T_{N}=0.99$, showing the computed distribution as a color map, with the wire frame showing the predicted two-sublattice Boltzmann probability distribution.

such coupling terms between the ferromagnetic spins and one or both antiferromagnetic sublattices, uncompensated spins as well as compensated spins ${ }^{98}$ may be simulated. This subject, however, is beyond the scope of the current work and will be addressed in a separate publication.

\section{PERFORMANCE AND BENCHMARKING}

Large-scale micromagnetics simulations require significant computational resources. An important advancement is the use of GPUs, which result in significant speed-up factors compared to CPUs, ${ }^{2}$ typically over an order of magnitude. All the computational routines in Boris may be executed on the CPU as well as on the GPU, either with single or double floating point precision. For CPU computations, Boris has been designed to run on shared memory devices with parallelization based on OpenMP. Fast Fourier transforms (FFTs) are computed using the FFTW3 library, ${ }^{32}$ configured to use threading. For GPU computations, Boris uses the CUDA toolkit, ${ }^{31}$ including for FFTs. Currently, true distributed computing in a cluster is not possible with Boris, although in a future work the possibility of using multiple GPUs for a single simulation will be explored. It should be noted that finite element/boundary element method codes are available for distributed computations, particularly suitable for curved geometries due to increased accuracy compared to finite difference codes, ${ }^{99}$ for example, Magpar has shown near-linear performance scaling. ${ }^{100}$ Boris can be controlled by using remote Python scripts; thus, execution and control of multiple independent remote Boris instances (for example, in a Linux cluster) is possible.

By far, the most expensive term to evaluate is the demagnetizing field, which involves evaluating a convolution sum over the entire mesh-see Eq. (4) - and normally takes $75 \%$ or more of the computation time in each iteration. The convolution sum may be 
evaluated very efficiently using the convolution theorem: a (2)3D FFT algorithm is used on the input magnetization; this is then multiplied with the demagnetizing kernel in the transform space, and an inverse (2)3D FFT algorithm is used to obtain the output demagnetizing field. The computational complexity of this approach increases as $N \log (N)$, compared to $N^{2}$ for the naïve evaluation of the convolution sum; thus, several orders of magnitude improvement may be achieved for a large number of computational cells $N$. The simplest method of implementing the 3D convolution algorithm consists of computing 1D FFTs along the $\mathrm{x}, \mathrm{y}$, and $\mathrm{z}$ directions, performing the point-by-point multiplication, then computing the $\mathrm{z}, \mathrm{y}$, and $\mathrm{x}$ inverse $1 \mathrm{D}$ FFTs in this order. With CUDA $^{31}$ implementations of GPU computations, these seven steps are most easily implemented using separate CUDA kernel launches. In particular, the $\mathrm{z}$ FFTs, point-by-point multiplications and $\mathrm{z}$ inverse FFTs are done in three separate steps. This can be inefficient for a small number of cells along the $\mathrm{z}$ direction. In Boris, a new approach is taken, termed pipelined convolution, where the $\mathrm{z}$ (inverse) FFTs and point-by-point multiplications are done using a single CUDA kernel launch, simultaneously for all three vector components. This involves manually coding the FFT algorithm and results in significant performance improvement over the nonpipelined approach due to more efficient use of GPU instruction bandwidth, up to a certain number of computational cells along the $\mathrm{z}$ direction.

To test the efficient implementation of computational routines in Boris, benchmark comparisons with Mumax $3^{2}$ have been performed. A testing platform consisting of a GTX $980 \mathrm{Ti}$ GPU in single floating precision mode on Ubuntu 20.04 was used. An identical simulation was configured for both programs, consisting in computing the magnetization response to a perpendicular magnetic field, with effective field contributions of demagnetizing field, exchange interaction, and applied field. The RK4 evaluation method was used, and the time per evaluation was measured, noting the RK4 method consists of four evaluations per time step iteration. The benchmarking scripts for both programs are available in the Boris GitHub repository. ${ }^{29}$ Typical results are shown in Fig. 12(a), for both 2D and 3D modes, showing the time per evaluation as a function of a total number of simulation cells $N$. In the $2 \mathrm{D}$ mode, the computational performance of Boris and Mumax 3 is comparable; however, in the $3 \mathrm{D}$ mode Boris is found to run significantly faster. More information is obtained by plotting the speedup factor $\left(t_{\mathrm{Mumax} 3} / t_{\mathrm{Boris}}\right)$ as a function of number of $z$ cells for a wide range of total number of computational cells, shown in Fig. 12(b). The pipelined convolution algorithm has been implemented up to $2^{4}$ cells along the $\mathrm{z}$ direction, thus for FFTs of up to 32 points, noting the circular convolution theorem requires doubling the input data size by zero padding when not using periodic boundary conditions. This approach is found to be significantly faster compared to Mumax3, with the speedup factor also increasing with the total number of computational cells. Above $2^{4}$ cells along the $\mathrm{z}$ direction, the pipelined convolution algorithm becomes less efficient than the non-pipelined algorithm; thus, $2^{4}$ is the largest value for which Boris implements the pipelined convolution approach, although speedup factors above 1 are still obtained for the non-pipelined convolution mode in all $3 \mathrm{D}$ cases.
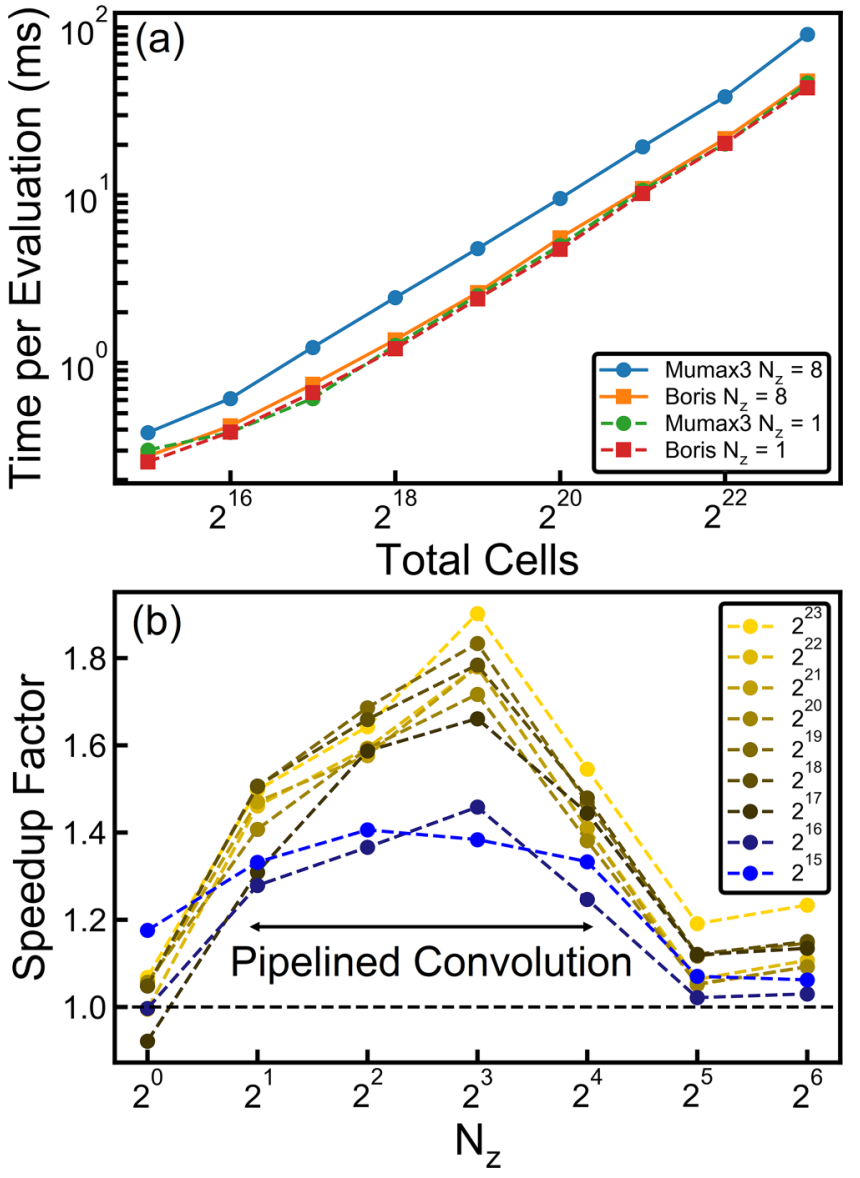

FIG. 12. Comparison of computational performance with Mumax3, for single floating point precision CUDA computations, benchmarked on a GTX $980 \mathrm{Ti}$ GPU under Ubuntu 20.04. (a) Time per evaluation as a function of a total number of simulation cells for $N_{z}=1$ ( $2 \mathrm{D}$ mode) and $N_{z}=8$ (3D mode). (b) Speedup factor, defined as the ratio of time per evaluation as $t_{\text {Mumax } 3} / t_{\text {Boris }}$, as a function of $N_{z}$ and a total number of computational cells. In Boris, 3D computations up to 16 cells along the $z$ direction $(80 \mathrm{~nm}$ thickness for a $5 \mathrm{~nm}$ cellsize) are handled using an efficient pipelined convolution algorithm, resulting in significant speedup factors compared to non-pipelined convolution in Mumax3, up to nearly twice faster on this platform for large simulations containing over $8 \times 10^{6}$ computational cells.

Finally, we test the performance scaling at fixed problem sizes for different GPUs as a function of available floating point operations per second (Flops), using the same simulation procedure explained above. The results are shown in Fig. 13 for the GTX 1050 Ti, GTX 980 Ti, RTX 2080 Super, and RTX $2080 \mathrm{Ti}$ GPUs, using GTX $1050 \mathrm{Ti}$ as a reference. Normally strong scaling as a function of number of available processors is described by Amdahl's law; ${ }^{101}$ however, for the results in Fig. 13, where not only the number of CUDA cores changes, but also the operating frequency, memory bandwidth, and microarchitecture changes, we simply indicate the ideal scaling of 1:1 increase with 


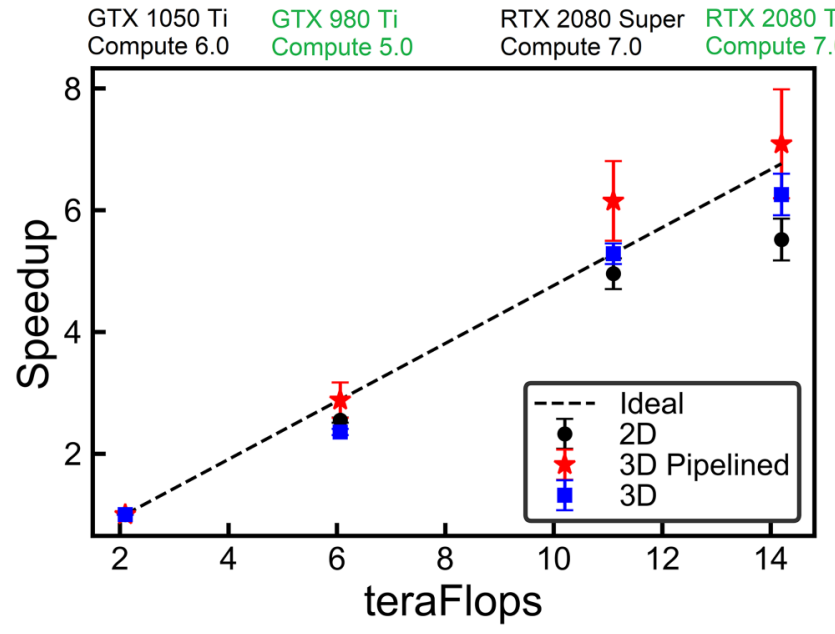

FIG. 13. Scaling at fixed problem size as a function of available single precision (FP32) floating point operations per second (Flops), across three different CUDA Compute major versions $(5.0,6.0,7.0)$, for the $2 \mathrm{D}$, pipelined $3 \mathrm{D}$, and standard 3D simulation modes. The GTX $1050 \mathrm{Ti}$ GPU was taken as a reference when computing the speedup factors, and these have been averaged for fixed problem sizes with $2^{20}, 2^{21}, 2^{22}$, and $2^{23}$ number of simulation cells.

available Flops for comparison. The performance increase is comparable to the ideal case on average. We note the most recent Ampere microarchitecture, with CUDA Compute 8.0, has GPUs with available Flops in the range of 20-40 teraFlops; however, we have not tested Boris in this range yet, although the code can also be compiled on this microarchitecture.

\section{CONCLUSIONS AND OUTLOOK}

Here, we have presented the main mathematical models implemented and testing of a new comprehensive computational magnetism research software. This represents a significant addition to the body of modeling capabilities introduced in other comparable open-source software, including OOMMF, ${ }^{1}$ Mumax $3,{ }^{2}$ and Fidimag. ${ }^{3}$ Thus, in addition to the existing micromagnetics modeling software, a new multi-mesh modeling paradigm is introduced, allowing complex simulations with multiple independently discretized computational meshes and materials. This allows simulations of multi-material structures, including ferromagnetic, antiferromagnetic, ferrimagnetic, and nonmagnetic and substrate materials, without the constraint of fitting the computations on a single uniformly discretized finite difference mesh, while still preserving the computational performance associated with finite difference methods. In addition to magnetization dynamics models, including LLG, LLB, and stochastic and two-sublattice models, Boris also implements a drift-diffusion spin transport solver in ferromagnetic materials, as well as a heat flow solver in multi-layered structures.

While the implemented spin transport solver is only applicable to ferromagnetic materials, a future development consists in extending the drift-diffusion model implementation to a twosublattice model, for example, as introduced in Ref. 102 with appropriate boundary conditions. Magnetoelastic effects may be modeled in Boris either using a uniform stress or by importing an externally computed strain or mechanical displacement, similar to the approach implemented in an OOMMF extension. ${ }^{103} \mathrm{~A}$ future development will implement both a multi-layered elastostatics solver and an elastodynamics solver, ${ }^{104}$ allowing complex simulations with non-uniform and time-dependent strains, including magnetoelastic and magnetostriction-related dynamical effects. Finally, a basic atomistic modeling ${ }^{105}$ capability has already been introduced in Boris, with a view to implementing true multi-scale simulations ${ }^{106,107}$ in the multi-mesh paradigm, although this was not discussed in the current work and will be treated in a separate publication.

\section{ACKNOWLEDGMENTS}

The author would like to thank Callum Robert Mackinnon for help with benchmarking the performance scaling of Boris.

\section{APPENDIX A: MICROMAGNETICS EFFECTIVE FIELD TERMS}

Effective field terms for various interactions are included as additive terms in $\mathbf{H}_{\text {eff, }}$, in either the LLG or LLB equations. These are usually obtained from their corresponding energy density terms using the relation $\mathbf{H}=\frac{-1}{\mu_{0} M_{s}} \frac{\partial \varepsilon}{\partial \mathbf{m}}$. The main terms implemented in Boris not already given in the main text are shown in Table I. Parameter definitions are not repeated here if given in the main text.

\section{APPENDIX B: LANDAU-LIFSHITZ-BLOCH TEMPERATURE DEPENDENCES}

For the two-sublattice model, we introduce convenient dimensionless micromagnetic parameters $\tau_{i}$ and $\tau_{i j} \in[0,1]$. These are coupling parameters between exchange parameters and the phase transition temperature, where $\left|J_{0, \mathrm{i}}\right|=3 \tau_{i} k_{B} T_{N},\left|J_{0, \mathrm{ij}}\right|=3 \tau_{i j} k_{B} T_{N}$. Here, $J_{0, i(j)}$ is the effective exchange parameter for intra-lattice $(i=A, B)$ and inter-lattice $(i, j=A, B, i \neq j)$ coupling, respectively, obtained from the exchange parameters as given in Ref. 94. For a simple prototypical antiferromagnet, we have $\tau_{A}=\tau_{B}=\tau_{A B}=\tau_{B A}=0.5 \quad\left(J_{0, \mathrm{ij}}<0\right)$. For $\tau_{A}=1, \tau_{B}=0, \tau_{A B}=\tau_{B A}=0$, the temperature dependences given below, as well as the two-sublattice LLB equation, reduce to the ferromagnetic LLB case. Thus, here we give the general case in terms of $\tau$ parameters.

The damping parameters are continuous at $T_{N}$-the phase transition temperature-and are given by

$$
\begin{aligned}
& \alpha_{\perp, i}=\alpha_{i}\left(1-\frac{T}{3\left(\tau_{i}+\tau_{i j} m_{e, j} / m_{e, i}\right) \tilde{T}_{N}}\right), \quad T<T_{N}, \\
& \alpha_{\|, i}=\alpha_{i}\left(\frac{2 T}{3\left(\tau_{i}+\tau_{i j} m_{e, j} / m_{e, i}\right) \tilde{T}_{N}}\right), \quad T<T_{N}, \\
& \alpha_{\perp, i}=\alpha_{\|, i}=\frac{2 T}{3 T_{N}}, \quad T \geq T_{N} .
\end{aligned}
$$


TABLE I. Effective field terms implemented in Boris, not already given in the main text.

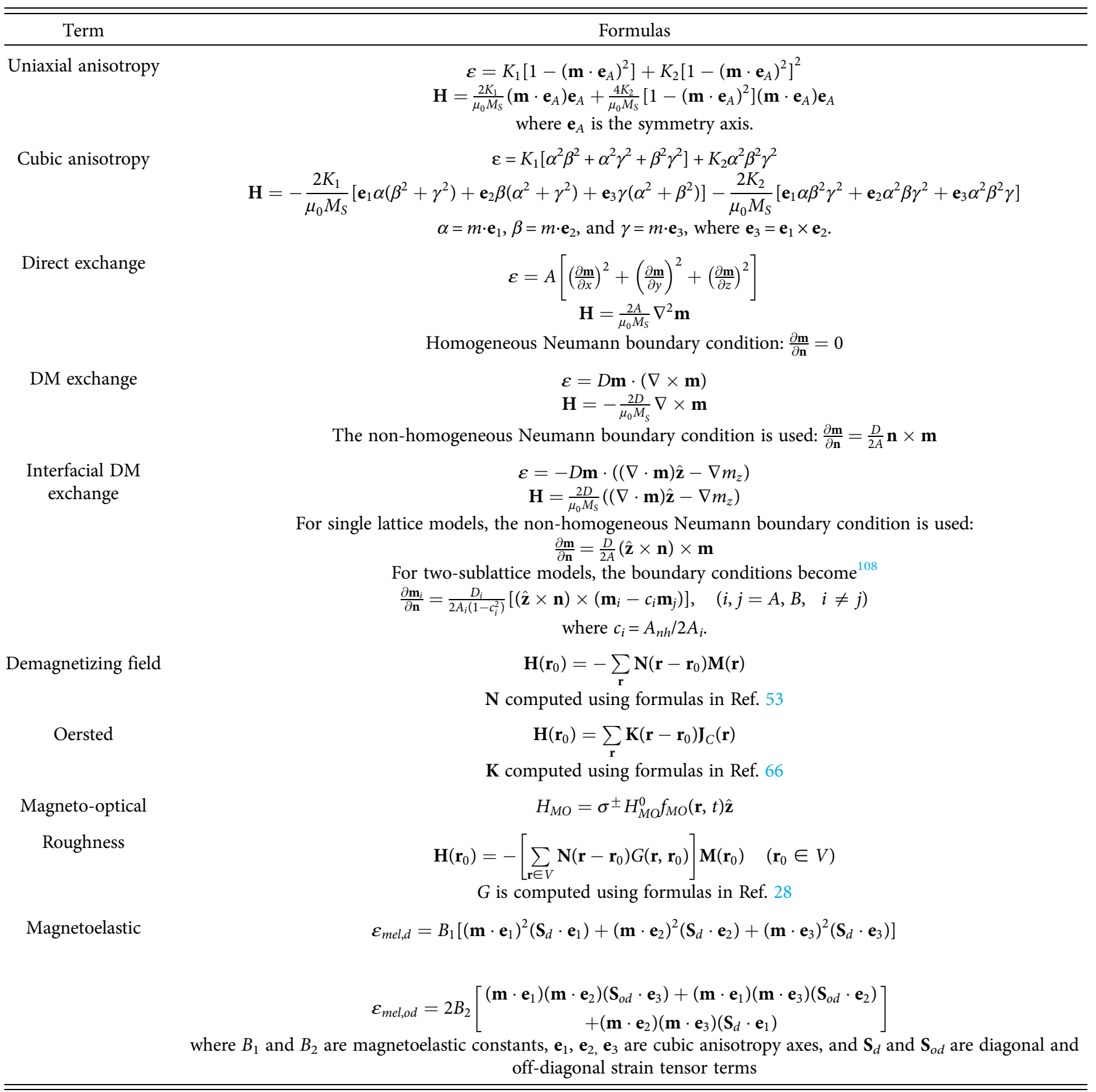

We denote $\tilde{T}_{N}$ the re-normalized transition temperature as

$$
\tilde{T}_{N}=\frac{2 T_{N}}{\tau_{A}+\tau_{B}+\sqrt{\left(\tau_{A}-\tau_{B}\right)^{2}+4 \tau_{A B} \tau_{B A}}} .
$$

The normalized equilibrium magnetization functions $m_{e, i}$ are obtained from the Curie-Weiss law as

$$
m_{e, i}=B\left[\left(m_{e, i} \tau_{i}+m_{e, j} \tau_{i j}\right) 3 \tilde{T}_{N} / T+\mu_{i} \mu_{0} H_{e x t} / k_{B} T\right],
$$


(a)

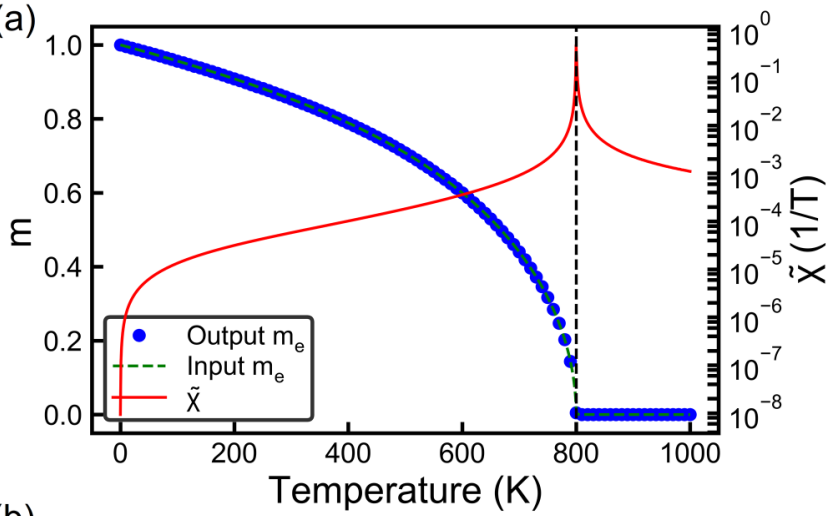

(b)
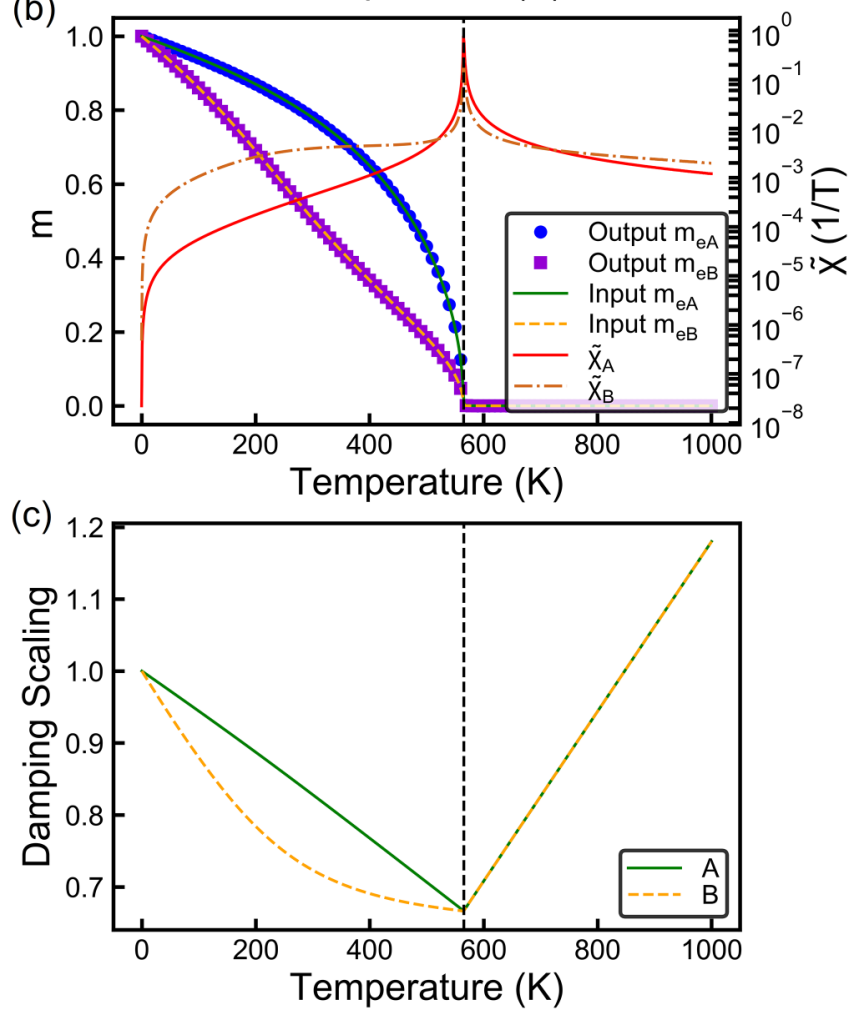

FIG. 14. Temperature dependences of magnetization scaling functions and relative longitudinal susceptibilities, showing both the model input functions and output computed functions for (a) one lattice LLB model with $T_{C}=800 \mathrm{~K}, \mu=1 \mu_{B}$ and (b) two-sublattice LLB model with parameters obtained from Ref. 94 for $\mathrm{Gd}_{25}(\mathrm{FeCo})_{75}$, namely, $T_{C}=565 \mathrm{~K}, \mu_{A}=\mu_{\mathrm{FeCo}}$ $=1.92 \mu_{B}, \mu_{B}=\mu_{\mathrm{Gd}}=7.63 \mu_{B}, \tau_{A}=0.958, \tau_{B}=0.127, \tau_{A B}=0.111, \tau_{B A}=0.333$. (c) Damping scaling functions for the two-sublattice model with $\mathrm{Gd}_{25}(\mathrm{FeCO})_{75}$ parameters.

where $B(x)=\operatorname{coth}(x)-1 / x$ and $\mu_{i}$ is the atomic magnetic moment.

The longitudinal relaxation field that includes both intralattice and inter-lattice contributions is given by

$$
\begin{aligned}
\mathbf{H}_{\|, i}= & \left\{\frac{1}{2 \mu_{0} \tilde{\chi}_{\|, i}}\left(1-\frac{m_{i}^{2}}{m_{e, i}^{2}}\right)+\frac{3 \tau_{i j} k_{B} T_{N}}{2 \mu_{0} \mu_{i}}\right. \\
& \left.\times\left[\frac{\tilde{\chi}_{\|, j}}{\tilde{\chi}_{\|, i}}\left(1-\frac{m_{i}^{2}}{m_{e, i}^{2}}\right)-\frac{m_{e, j}}{m_{e, i}}\left(\hat{\mathbf{m}}_{i} \cdot \hat{\mathbf{m}}_{j}\right)\left(1-\frac{m_{j}^{2}}{m_{e, j}^{2}}\right)\right]\right\} \mathbf{m}_{i}, T<T_{N}, \\
\mathbf{H}_{\|, i}= & -\left\{\frac{1}{\mu_{0} \tilde{\chi}_{\|, i}}+\frac{3 \tau_{i j} k_{B} T_{N}}{\mu_{0} \mu_{i}}\left[\frac{\tilde{\chi}_{\|, j}}{\tilde{\chi}_{\|, i}}-\frac{m_{e, j}}{m_{e, i}}\left(\hat{\mathbf{m}}_{i} \cdot \hat{\mathbf{m}}_{j}\right)\right]\right\} \mathbf{m}_{i}, T>T_{N} .
\end{aligned}
$$

Here, $\hat{\mathbf{m}}_{i}=\mathbf{m}_{i} / m_{i}$, and the relative longitudinal susceptibility is given in Eq. (24).

Similarly to the ferromagnetic case, the default magnetization temperature scaling is given as $M_{e, i}=m_{e, i} M_{S, i}^{0}$. The anisotropy constant follows the temperature dependence $K_{1, i}=K_{1, i}^{0} m_{e, i}^{3}$. The intra-lattice exchange stiffness $A_{i}$ has the temperature dependence $A_{i}=A_{i}^{0} m_{e, i}^{2}$, while the inter-lattice exchange stiffnesses have the temperature dependences $A_{h(n h), i}=A_{h(n h), i}^{0} m_{e, i} m_{e, j}$. The DMI exchange parameter follows the temperature dependence $D_{i}=D_{i}^{0} m_{e, i}^{2}$. Note these temperature dependences can be adjusted depending on the material simulated where appropriate, for example, using $m_{e, i}$ exponents computed using an atomistic model.

We further show examples of magnetization temperature scaling laws as well as relative longitudinal susceptibility temperature dependences in Fig. 14. In Fig. 14(a), we take a simple ferromagnet with $\mu=1 \mu_{B}$ and $T_{C}=800 \mathrm{~K}$, plotting the computed relative susceptibility above and below $T_{C}$ based on Eq. (16). The input $m_{e}$ function-Eq. (15) - is also plotted for an applied external field of $10 \mathrm{kA} / \mathrm{m}$, and we test the correct implementation by computing the output magnetization temperature dependence using the LLB equation; as expected the input and output $m_{e}$ scalings are virtually identical. Next, we consider the two-sublattice LLB model using the parameters for $\mathrm{Gd}_{25}(\mathrm{FeCo})_{75}$ given in Ref. 94. From these, we obtain the dimensionless coupling parameters $\tau_{A}=0.958$, $\tau_{B}=0.127, \tau_{A B}=0.111$, and $\tau_{B A}=0.333$ for $T_{C}=565 \mathrm{~K}$. Again, we plot the relative longitudinal susceptibilities-Eq. (24) - for the two sublattices $(A=\mathrm{FeCo}, B=\mathrm{Gd})$ in Fig. 14(b), as well as the input $m_{e, i}$ functions from Eq. (B3) - as for the ferromagnetic case, these are virtually identical to the magnetization temperature scaling computed using the two-sublattice LLB model as expected. The damping parameter temperature scaling functions-Eq. (B1)-are plotted in Fig. 14(c).

\section{DATA AVAILABILITY}

The data that support the findings of this study are available from the corresponding author upon reasonable request and the scripts used to obtain the data are available at https://github.com/ SerbanL/Boris2/tree/master/BorisArticle Ref. 29.

\section{REFERENCES}

${ }^{1}$ M. J. Donahue and D. G. Porter, OOMMF User's Guide, Version 1.0. Interagency Report NISTIR 6376, 1999.

${ }^{2}$ A. Vansteenkiste, J. Leliaert, M. Dvornik, M. Helsen, F. Garcia-Sanchez, and B. Van Waeyenberge, "The design and verification of Mumax3," AIP Adv. 4, 107133 (2014). 
${ }^{3}$ M.-A. Bisotti, D. Cortés-Ortuño, R. Pepper, W. Wang, M. Beg, T. Kluyver, and H. Fangohr, "Fidimag-A finite difference atomistic and micromagnetic simulation package," J. Open Res. Softw. 6, 22 (2018).

${ }^{4}$ J. Leliaert and J. Mulkers, "Tomorrow's micromagnetic simulations," J. Appl, Phys. 125, 180901 (2019).

${ }^{5}$ T. L. Gilbert, "A Lagrangian formulation of the gyromagnetic equation of the magnetic field," Phys. Rev. 100, 1243 (1955).

${ }^{6}$ D. A. Garanin, "Fokker-Planck and Landau-Lifshitz-Bloch equations for classical ferromagnets," Phys. Rev. B 55, 3050 (1997).

${ }^{7}$ D. A. Garanin and O. Chubykalo-Fesenko, "Thermal fluctuations and longitudinal relaxation of single-domain magnetic particles at elevated temperatures," Phys. Rev. B 70, 212409 (2004).

${ }^{8}$ R. F. L. Evans et al., "Stochastic form of the Landau-Lifshitz-Bloch equation," Phys. Rev. B 85, 014433 (2012).

${ }^{9} \mathrm{~S}$. Zhang and Z. Li, "Roles of nonequilibrium conduction electrons on the magnetization dynamics of ferromagnets," Phys. Rev. Lett. 93, 127204 (2004).

${ }^{10} \mathrm{~S}$. Lepadatu, "Effect of inter-layer spin diffusion on skyrmion motion in magnetic multilayers," Sci. Rep. 9, 9592 (2019).

${ }^{11}$ C. R. MacKinnon, S. Lepadatu, T. Mercer, and P. R. Bissell, "Role of an additional interfacial spin-transfer torque for current-driven skyrmion dynamics in chiral magnetic layers," Phys. Rev. B 102, 214408 (2020).

${ }^{12}$ M. I. Dyakonov and V. I. Perel, "Possibility of orienting electron spins with current," JETP Lett. 13, 467-469 (1971).

${ }^{13}$ J. C. Slonczewski, "Current-driven excitation of magnetic multilayers," J. Magn. Magn. Mater. 159, L1-L7 (1996).

${ }^{14} \mathrm{M}$. I. Dyakonov, "Magnetoresistance due to edge spin accumulation," Phys. Rev. Lett. 99, 126601 (2007).

${ }^{15}$ S. Zhang, P. M. Levy, and A. Fert, "Mechanisms of spin-polarized currentdriven magnetization switching," Phys. Rev. Lett. 88, 236601 (2002).

${ }^{16}$ A. Brataas, Y. U. V. Nazarov, and G. E. W. Bauer, "Finite-element theory of transport in ferromagnet-normal metal systems," Phys. Rev. Lett. 84, 2481 (2000).

${ }^{17}$ S. Lepadatu, "Unifed treatment of spin torques using a coupled magnetisation dynamics and three-dimensional spin current solver," Sci. Rep. 7, 12937 (2017).

18. E. Hirsch, "Spin Hall effect," Phys. Rev. Lett. 83, 1834 (1999).

${ }^{19}$ Y. Tserkovnyak, A. Brataas, and G. E. W. Bauer, "Enhanced Gilbert damping in thin ferromagnetic films," Phys. Rev. Lett. 88, 117601 (2002).

${ }^{\mathbf{2 0}} \mathrm{Y}$. Tserkovnyak and M. Mecklenburg, "Electron transport driven by nonequilibrium magnetic textures," Phys. Rev. B 77, 134407 (2008).

${ }^{21}$ S. Zhang and S. S.-L. Zhang, "Generalization of the Landau-Lifshitz-Gilbert equation for conducting ferromagnets," Phys. Rev. Lett. 102, 086601 (2009).

${ }^{\mathbf{2 2}}$ S. Lepadatu, "Interaction of magnetization and heat dynamics for pulsed domain wall movement with Joule heating," J. Appl. Phys. 120, 163908 (2016).

${ }^{23} \mathrm{U}$. Atxitia and O. Chubykalo-Fesenko, "Ultrafast magnetization dynamics rates within the Landau-Lifshitz-Bloch model," Phys. Rev. B 84, 144414 (2011).

${ }^{24} \mathrm{~S}$. Lepadatu, "Efficient computation of demagnetizing fields for magnetic multilayers using multilayered convolution," J. Appl. Phys. 126, 103903 (2019).

${ }^{25}$ I. Dzyaloshinsky, "A thermodynamic theory of "weak" ferromagnetism of antiferromagnetics," J. Phys. Chem. Solids 4, 241-255 (1958).

${ }^{\mathbf{2 6}}$ T. Moriya, "Anisotropic superexchange interaction and weak ferromagnetism," Phys. Rev. 120, 91 (1960).

${ }^{27} \mathrm{~B}$. Heinrich et al., "Bilinear and biquadratic exchange coupling in bcc $\mathrm{Fe} / \mathrm{Cu} /$ Fe trilayers: Ferromagnetic-resonance and surface magneto-optical Kerr-effect studies," Phys. Rev. B 47, 5077 (1993).

${ }^{\mathbf{2 8}}$ S. Lepadatu, "Effective field model of roughness in magnetic nano-structures," J. Appl. Phys. 118, 243908 (2015).

${ }^{29}$ See https://github.com/SerbanL/Boris2 for source code repository; accessed 3 August 32020 .

${ }^{30}$ User manual, https://www.researchgate.net/publication/331715880_Boris_ Computational_Spintronics_User_Manual, DOI:10.13140/RG.2.2.31496.88322/1; accessed 3 August 2020

${ }^{31}$ J. Nickolls, I. Buck, M. Garland, and K. Skadron, "Scalable parallel programming with CUDA," ACM Queue 6, 40-53 (2008).
${ }^{32} \mathrm{M}$. Frigo and S. G. Johnson, "The design and implementation of FFTW3," Proc. IEEE 93, 216-231 (2005).

${ }^{33} \mathrm{See}$ http://www.boris-spintronics.uk/online-materials-database/ for material parameters; accessed 3 August 2020.

${ }^{34}$ T. L. Gilbert, "A phenomenological theory of damping in ferromagnetic materials," IEEE Trans. Magn. 40, 3443 (2004)

${ }^{35}$ L. Exl et al., "Labonte's method revisited: An effective steepest descent method for micromagnetic energy minimization," J. Appl. Phys. 115, 17D118 (2014).

${ }^{36}$ J. Barzilai and J. M. Borwein, "Two-point step size gradient methods," IMA J. Numer. Anal. 8, 141-148 (1988).

${ }^{37}$ See https://www.ctcms.nist.gov/ rdm/std4/spec4.html for NIST micromagnetic modeling activity group ( $\mu \mathrm{MAG})$ website; accessed 22 July 2020.

${ }^{38} \mathrm{G}$. Venkat et al., "Proposal for a standard micromagnetic problem: Spin wave dispersion in a magnonic waveguide," IEEE Trans. Mag. 49, 524-529 (2013).

${ }^{39}$ K. M. Lebecki, M. J. Donahue, and M. W. Gutowski, "Periodic boundary conditions for demagnetization interactions in micromagnetic simulations," J. Phys. D Appl. Phys. 41, 175005 (2008).

${ }^{40} \mathrm{Z}$. Li and S. Zhang, "Domain-wall dynamics driven by adiabatic spin-transfer torques," Phys. Rev. B 70, 024417 (2004)

${ }^{41}$ S. Lepadatu, "Interaction of magnetization and heat dynamics for pulsed domain wall movement with Joule heating," J. Appl. Phys. 120, 163908 (2016).

${ }^{42}$ A. Thiaville, Y. Nakatani, J. Miltat, and Y. Suzuki, "Micromagnetic understanding of current-driven domain wall motion in patterned nanowires," Europhys. Lett. 69, 990-996 (2005).

${ }^{43}$ See https://www.ctcms.nist.gov/ rdm/std5/spec5.xhtml for NIST micromagnetic modeling activity group ( $\mu \mathrm{MAG})$ website; accessed 22 July 2020.

${ }^{44}$ R. F. Wang et al., "Artificial 'spin ice' in a geometrically frustrated lattice of nanoscale ferromagnetic islands," Nature 439, 303-306 (2006).

${ }^{45}$ J. P. Morgan, A. Stein, S. Langridge, and C. H. Marrows, "Thermal groundstate ordering and elementary excitations in artificial magnetic square ice," Nat. Phys. 7, 75-79 (2011).

${ }^{46} \mathrm{~S}$. Woo et al., "Observation of room-temperature magnetic skyrmions and their current driven dynamics in ultra-thin metallic ferromagnets," Nat. Mater. 15, 501-506 (2016).

${ }^{47} \mathrm{~W}$. Legrand et al., "Room-temperature current-induced generation and motion of sub100 nm skyrmions," Nano Lett. 17, 2703-2712 (2017).

${ }^{48} \mathrm{~K}$. Litzius et al., "Skyrmion Hall effect revealed by direct time-resolved X-ray microscopy,” Nat. Phys. 13, 170-175 (2017)

${ }^{49} \mathrm{~A}$. Hrabec et al., "Current-induced skyrmion generation and dynamics in symmetric bilayers," Nat. Commun. 8, 15765 (2017).

${ }^{50}$ S. Lepadatu et al., "Very low critical current density for motion of coupled domain walls in synthetic ferrimagnet nanowires," Sci. Rep. 7, 1640 (2017).

${ }^{51}$ A. Hrabec et al., "Velocity enhancement by synchronization of magnetic domain walls," Phys. Rev. Lett. 120, 227204 (2018).

${ }^{52}$ S.-H. Yang, K.-S. Ryu, and S. Parkin, "Domain-wall velocities of up to 750 $\mathrm{ms}^{-1}$ driven by exchange-coupling torque in synthetic antiferromagnets," Nat. Nanotechnol. 10, 221-226 (2015).

${ }^{53}$ A. J. Newell, W. Williams, and D. J. Dunlop, "A generalization of the demagnetizing tensor for nonuniform magnetization,” J. Geophys. Res. Solid Earth 98, 9551-9555, (1993).

${ }^{54}$ H. Fangohr, G. Bordignon, M. Franchin, A. Knittel, P. A. J. de Groot, and T. Fischbacher, "A new approach to (quasi) periodic boundary conditions in micromagnetics: The macrogeometry," J. Appl. Phys. 105, 07D529 (2009).

${ }^{55} \mathrm{M}$. A. Ruderman and C. Kittel, "Indirect exchange coupling of nuclear magnetic moments by conduction electrons," Phys. Rev. 96, 99-102 (1954).

${ }^{56} \mathrm{~T}$. Kasuya, "A theory of metallic ferro- and antiferromagnetism on Zener's model," Prog. Theor. Phys. 16, 45-57 (1956).

${ }^{57} \mathrm{~K}$. Yosida, "Magnetic properties of Cu-Mn alloys," Phys. Rev. 106, 893 (1957).

${ }^{58}$ M. M. Vopson, J. Naylor, T. Saengow, E. G. Rogers, S. Lepadatu, and Y. K. Fetisov, "Development of flexible Ni80Fe20 magnetic nano-thin films," Phys. B Condens. Matter 525, 12 (2017). 
${ }^{\mathbf{5 9}} \mathrm{M}$. Belusky, S. Lepadatu, J. Naylor, and M. M. Vopson, "Evidence of substrate roughness surface induced magnetic anisotropy in Ni80Fe20 flexible thin films," J. Magn. Magn. Mater. 478, 77 (2019).

${ }^{60}$ M. Belusky, S. Lepadatu, J. Naylor, and M. M. Vopson, "Study of roughness effect in Fe and Co thin films prepared by plasma magnetron sputtering," Physica B 574, 411666 (2019).

${ }^{61}$ M. M. Vopson and S. Lepadatu, "Solving the electrical control of magnetic coercive field paradox," Appl. Phys. Lett. 105, 122901 (2014).

${ }^{62}$ S. Lepadatu and M. Vopson, "Heat assisted multiferroic solid-state memory," Materials 10, 991 (2017).

${ }^{63}$ T. Chen et al., "Spin-torque and spin-Hall nano-oscillators," Proc. IEEE 104, 1919-1945 (2016)

64. Åkerman, “Toward a universal memory," Science 308, 508-510 (2005).

${ }^{65}$ J. W. Thomas, Numerical Partial Differential Equations: Finite Difference Methods (Springer-Verlag, 1995).

${ }^{66} \mathrm{~B}$. Krüger, "Current-driven magnetization dynamics: analytical modeling and numerical simulation," Ph.D. dissertation, University of Hamburg, 2011, Appendix D, p. 118.

${ }^{67} \mathrm{C}$. Abert et al., "A three-dimensional spin-diffusion model for micromagnetics," Sci. Rep. 5, 14855 (2015).

${ }^{68}$ D. Claudio-Gonzalez, A. Thiaville, and J. Miltat, "Domain wall dynamics under nonlocal spin-transfer torque," Phys. Rev. Lett. 108, 227208 (2012).

${ }^{69} \mathrm{~A}$. Bisig et al., "Enhanced nonadiabaticity in vortex cores due to the emergent Hall effect," Phys. Rev. Lett. 117, 277203 (2016).

${ }^{70} \mathrm{~K}$. Ando et al., "Electric manipulation of spin relaxation using the spin Hall effect," Phys. Rev. Lett. 101, 036601 (2008).

${ }^{71}$ J. Xiao, A. Zangwill, and M. D. Stiles, "Boltzmann test of Slonczewski's theory of spin-transfer torque," Phys. Rev. B 70, 172405 (2004).

${ }^{72}$ J. Xiao, A. Zangwill, and M. D. Stiles, "Macrospin models of spin transfer dynamics," Phys. Rev. B 72, 014446 (2005).

${ }^{73}$ J. L. García-Palacios and F. J. Lázaro, "Langevin-dynamics study of the dynamical properties of small magnetic particles," Phys. Rev. B 58, 14937 (1998).

${ }^{74}$ U. Atxitia, D. Hinzke, O. Chubykalo-Fesenko, U. Nowak, H. Kachkachi, O. N. Mryasov, R. F. Evans, and R. W. Chantrell, "Multiscale modeling of magnetic materials: Temperature dependence of the exchange stiffness," Phys. Rev. B 82, 134440 (2010)

${ }^{75} \mathrm{~L}$. Rozsa, U. Atxitia, and U. Novak, "Temperature scaling of the Dzyaloshinsky-Moriya interaction in the spin wave spectrum," Phys. Rev. B 96, 094436 (2017).

${ }^{76} \mathrm{H}$. B. Callen and E. Callen, "The present status of the temperature dependence of magnetocrystalline anisotropy, and the power law," J. Phys. Chem. Sol. 27, 1271-1285 (1966).

${ }^{77}$ P. Asselin, R. F. L. Evans, J. Barker, R. W. Chantrell, R. Yanes, O. Chubykalo-Fesenko, D. Hinzke, and U. Nowak, "Constrained Monte Carlo method and calculation of the temperature dependence of magnetic anisotropy," Phys. Rev. B 82, 054415 (2010).

${ }^{78}$ R. Moreno, R. F. L. Evans, S. Khmelevskyi, M. C. Muñoz, R. W. Chantrell, and O. Chubykalo-Fesenko, "Temperature-dependent exchange stiffness and domain wall width in Co," Phys. Rev. B 94, 104433 (2016).

${ }^{79}$ R. Tomasello, K. Y. Guslienko, M. Ricci, A. Giordano, J. Barker, M. Carpentieri, O. Chubykalo-Fesenko, and G. Finocchio, "Origin of temperature and field dependence of magnetic skyrmion size in ultrathin nanodots," Phys. Rev. B 97, 060402 (2018).

${ }^{80} \mathrm{D}$. Hinzke and U. Nowak, "Domain wall motion by the magnonic spin Seebeck effect," Phys. Rev. Lett. 107, 027205 (2011).

${ }^{81}$ E. Beaurepaire, J.-C. Merle, A. Daunois, and J.-Y. Bigot, "Ultrafast spin dynamics in ferromagnetic nickel," Phys. Rev. Lett. 76, 4250 (1996).

${ }^{82} \mathrm{~B}$. Koopmans et al., "Explaining the paradoxical diversity of ultrafast laserinduced demagnetization," Nat. Mater. 9, 259 (2010).
${ }^{83} \mathrm{~N}$. Nagaosa and Y. Tokura, "Topological properties and dynamics of magnetic skyrmions," Nat. Nanotechnol. 8, 899 (2013).

${ }^{84}$ S.-G. Je et al., "Creation of magnetic skyrmion bubble lattices by ultrafast laser in ultrathin films," Nano Lett. 18, 7362 (2018).

${ }^{85}$ S. Lepadatu, "Emergence of transient domain wall skyrmions after ultrafast demagnetization," Phys. Rev. B 102, 094402 (2020).

${ }^{86}$ C. Marrows, "Addressing an antiferromagnetic memory," Science 351, 558 (2016).

${ }^{87}$ T. Jungwirth, X. Marti, P. Wadley, and J. Wunderlich, "Antiferromagnetic spintronics," Nat. Nanotechnol. 11, 231 (2016).

${ }^{88}$ T. Jungwirth, J. Sinova, A. Manchon, X. Marti, J. Wunderlich, and C. Felser, "The multiple directions of antiferromagnetic spintronics," Nat. Phys. 14, 200-203 (2018).

${ }^{89} \mathrm{P}$. Wadley et al., "Electrical switching of an antiferromagnet," Science 351, 587-590 (2016).

${ }^{90}$ S. Baierl et al., "Nonlinear spin control by terahertz-driven anisotropy fields," Nat. Photonics 10, 715-718 (2016).

${ }^{91}$ S. Woo et al., "Current-driven dynamics and inhibition of the skyrmion Hall effect of ferrimagnetic skyrmions in GdFeCo films," Nat. Commun. 9, 959 (2018).

${ }^{92}$ J. Nogués and I. K. Schuller, "Exchange bias," J. Magn. Magn. Mater. 192, 203-232 (1999).

${ }^{93}$ U. Atxitia, P. Nieves, and O. Chubykalo-Fesenko, "Landau-Lifshitz-Bloch equation for ferrimagnetic materials,” Phys. Rev. B 86, 104414 (2012).

${ }^{94}$ P. Nieves, U. Atxitia, R. W. Chantrell, and O. Chubykalo-Fesenko, "The classical two-sublattice Landau-Lifshitz-Bloch equation for all temperatures," Low Temp. Phys. 41, 739 (2015).

${ }^{95}$ F. Keefer and C. Kittel, "Theory of antiferromagnetic resonance," Phys. Rev. 85, 329 (1952).

${ }^{96}$ W. H. Meiklejohn and C. P. Bean, "New magnetic anisotropy," Phys. Rev. 102, 1413 (1956).

${ }^{97}$ M. M. Vopson, M. Belusky, and S. Lepadatu, "Diamagnetic coupling for magnetic tuning in nano-thin films," Apply. Phys. Lett. 116, 252402 (2020).

${ }^{98}$ R. L. Stamps, "Mechanisms for exchange bias," J. Phys. D Appl. Phys. 33, R247-R268 (2000)

${ }^{99}$ C. Abert, L. Exl, G. Selke, A. Drews, and T. Schrefl, "Numerical methods for the stray-field calculation: A comparison of recently developed algorithms," J. Magn. Magn. Mater. 326, 176-185 (2013).

${ }^{100}$ W. Scholz, J. Fidler, T. Schrefl, D. Suess, R. Dittrich, H. Forster, and V. Tsiantos, "Scalable parallel micromagnetic solvers for magnetic nanostructures," Comput. Mater. Sci. 28, 366 (2003).

${ }^{101} \mathrm{G}$. M. Amdahl, "Validity of the single processor approach to achieving large scale computing capabilities," in AFIPS Conference Proceedings (ACM Digital Library, 1967), Vol. 30, pp. 483-485.

${ }^{102}$ A. Manchon, "Spin diffusion and torques in disordered antiferromagnets," J. Phys. Condens. Matter 29, 104002 (2017).

${ }^{103}$ Y. Yahagi, B. Harteneck, S. Cabrini, and H. Schmidt, "Controlling nanomagnet magnetization dynamics via magnetoelastic coupling," Phys. Rev. B 90, 140405 (2014).

${ }^{104}$ A. G. Gurevich and G. A. Melkov, Magnetization Oscillations and Waves (CRC Press, 1996).

${ }^{105}$ R. F. L. Evans et al., "Atomistic spin model simulations of magnetic nanomaterials," J. Phys. Condens. Matter 26, 103202 (2014).

${ }^{106}$ A. De Lucia, B. Krüger, O. A. Tretiakov, and M. Kläui, "Multiscale model approach for magnetization dynamics simulations," Phys. Rev. B 94, 184415 (2016).

${ }^{107}$ É Méndez, M. Poluektov, G. Kreiss, O. Eriksson, and M. Pereiro, "Multiscale approach for magnetization dynamics: Unraveling exotic magnetic states of matter," Phys. Rev. Res. 2, 013092 (2020).

${ }^{108}$ L. Sánchez-Tejerina, V. Puliafito, P. K. Amiri, M. Carpentieri, and G. Finocchio, "Dynamics of domain-wall motion driven by spin-orbit torque in antiferromagnets," Phys. Rev. B 101, 014433 (2020). 